\title{
Optimal Testing Strategy for the Identification of COVID-19 Infections
}

\author{
Michail Chatzimanolakis ${ }^{1, *}$, Pascal Weber ${ }^{1, *}$, George Arampatzis ${ }^{1}$, \\ Daniel Wälchli ${ }^{1}$, Ivica Kičić ${ }^{1}$, Petr Karnakov ${ }^{1}$, Costas Papadimitriou ${ }^{2}$, \\ Petros Koumoutsakos ${ }^{1, \dagger}$ \\ ${ }^{1}$ Computational Science and Engineering Laboratory, ETH Zurich, \\ Clausiusstrasse 33, CH-8092, Switzerland \\ ${ }^{2}$ Department of Mechanical Engineering, University of Thessaly, \\ Pedion Areos, GR-38334 Volos, Greece \\ * These authors contributed equally to this work. \\ $\dagger$ Corresponding author. E-mail: petros@ethz.ch
}

The systematic identification of infectious, yet unreported, individuals is critical for the containment of the COVID-19 pandemic. We present a strategy for identifying the location, timing and extent of testing that maximizes information gain for such infections. The optimal testing strategy relies on Bayesian experimental design and forecasting epidemic models that account for time dependent interventions. It is applicable at the onset and spreading of the epidemic and can forewarn for a possible recurrence of the disease after relaxation of interventions. We examine its application in Switzerland and show that it can provide timely and systematic guidance for the effective identification of infectious individuals with finite testing resources. The methodology and the open source code are readily adaptable to countries around the world. We present a strategy for the optimal allocation of testing resources in order to detect COVID-19 infections in a country's population. 
medRxiv preprint doi: https://doi.org/10.1101/2020.07.20.20157818; this version posted July 27, 2020. The copyright holder for this preprint

\section{Introduction}

The identification of unreported individuals infected by the SARS-CoV-2 virus is critical for the quantification and forecasting of the COVID-19 pandemic (1-3). Presently the spread of the disease is quantified by the reported numbers of infections, hospitalizations, recoveries and deaths. In turn, these quantities inform epidemiology models that provide short term forecasts for the spread of the epidemic and help quantify the role of possible interventions. The veracity of these forecasts depends on the discrepancy between the numbers of reported and unreported, yet infectious, individuals. The estimation of such unreported infections has been the subject of important testing campaigns (4). A challenge for these campaigns is that their estimates rely on testing symptomatic individuals or individuals that have been selected based on certain a priori selected criteria (hospital visits, airport arrivals, geographic vicinity to researchers, etc.) as well as on randomized tests of the population (5). There is broad recognition that efficient testing strategies are critical for the timely identification of infectious individuals and the optimal allocation of resources (6-8). However, targeted testing entails bias while randomized tests require access to a high percentage of the population with commensurate high costs.

Here we present an Optimal Testing Strategy (OpTS) that provides maximum information gain over the prior knowledge regarding infections. The method employs forecasts by epidemiological models (9) with parameters that are adjusted as data become available through a Bayesian inference framework. The forecasts are combined with Bayesian experimental design (10-13) to determine optimal testing parameters. The resulting OpTS is applicable in all stages of the pandemic, regardless of the availability of data.

We employ the $S E I^{r} I^{u} R$ model (14) that predicts the number of susceptible $(S)$, exposed $(E)$, infectious reported $\left(I^{r}\right)$, unreported $\left(I^{u}\right)$, and removed $(R)$ individuals from the population.

The model amounts to a set of coupled ordinary differential equations with parameters inferred 
medRxiv preprint doi: https://doi.org/10.1101/2020.07.20.20157818; this version posted July 27, 2020. The copyright holder for this preprint

through a Bayesian framework. The $S E I^{r} I^{u} R$ models the spread of a disease in a country's population distributed in a number of communities that are interacting through mobility networks. Here we focus on Switzerland and consider its cantons as the respective communities. The model parameters are: the relative transmission rate $(\mu)$, the virus latency period $(Z)$, the infectious period $(D)$ and the reporting rate $(\alpha)$. Moreover the transmission rate $(\beta)$ and the mobility factor $(\theta)$ are considered to be time dependent in order to account for government interventions.

Bayesian inference is used to quantify and propagate uncertainties (15) in the model parameters. At the onset of the epidemic, the uncertainty is quantified solely through prior probability distributions. As daily infections data become available, the model is updated by estimating the posterior distribution of its parameters using Bayesian inference. These distributions are used to propagate uncertainties in the model forecasts, informing decision makers regarding the containment of the disease (9). We note that the uncertainty in the model parameters and initial conditions has a profound effect on predictions of the disease dynamics and associated uncertainty bounds (16). Large uncertainty around the most probable parameter values may result in large uncertainty bounds for critical quantities of interest, thus hindering the decision process for identifying effective interventions.

The OpTS aims to acquire the most informative data, to reduce model uncertainties, with limited test resources. Minimizing the uncertainty of the model parameters leads to more reliable predictions for quantities such as the reproduction number (17). Moreover, it results in state variables with smaller uncertainty bounds, thus reducing risks associated with the decision making process including timing, extent of interventions and probability of exceeding hospital capacity. Here the OpTS provides optimal estimates for times and selected communities to estimate the number of unreported infectious individuals in a country's (Switzerland) population. We measure the information gain from these tests using a utility function (18-21) based on the 
Kullback-Leibler divergence between the inferred posterior distribution and the current prior distribution of the model parameters. We remark that the prior can be formulated using the posterior distribution estimated from daily data of the infectious reported individuals up to the current date (see Materials and Methods). Hence, in any stage of the epidemic we can estimate the optimal time and location/community where testing has to be carried out to maximize the expected information gain regarding infections in a population.

We show the effectiveness of the proposed OpTS over non-specific, randomized testing of sub-populations. Furthermore, we demonstrate the capabilities of the method throughout the COVID-19 pandemic, from the onset of the epidemic to monitoring the effectiveness of interventions and forewarning for a possible second outbreak. Our results are based on the spread of the coronavirus disease in the cantons of Switzerland and an extended $S E I^{r} I^{u} R$ epidemiological model that accounts for time dependent mitigation strategies (22). However, the testing method is readily applicable to other countries and can readily accommodate different epidemiological models.

\section{Optimal Testing during the COVID-19 pandemic}

We present OpTS for three different stages of the epidemic: (i) starting phase (blue), (ii) containment after enforcement of interventions (red) and (iii) relaxing of interventions and monitoring for a possible second outbreak (green) (Fig.11). The strategy relies on Bayesian experimental design and can operate when no data are available (as in the start of the epidemic) as well as when data have been accumulated, as in the last two stages of the epidemic. Testing campaigns rely on acquiring randomized samples from a population. The collected data, together with epidemiological models, help determine quantities of interest, such as the basic reproduction number of the disease (17). By suitably adapting the testing campaign, the data can help reduce the model uncertainty, thus enabling accurate forecasts and improved estimates regarding the 
severity of an epidemic.

A testing campaign consists of a set $(s)$ of randomized tests $s_{i}=\left(k_{i}, t_{i}\right), i=1, \ldots M_{y}$ performed in location $k_{i}$ and on day $t_{i}$. The expected information gain of a particular strategy for selecting the testing locations/times $s$ is quantified by using a utility function $\hat{U}(s)(18)$. The maximum of this function corresponds to an optimal strategy that yields the most information about the quantities of interest. A key challenge for OpTS is their computational cost (12). Here, the OpTS is rendered computationally efficient through a sequential optimization algorithm (23) that adds tests iteratively.

The OpTS relies the characterization and prediction of the spread of the disease based on forecasts by suitable epidemiological models (22). In turn, these forecasts rely on prior information and their predictions are further adjusted as data become available in a Bayesian inference framework (9). Here, the set of Ordinary Differential Equations (ODEs) describing the $S E I^{r} I^{u} R$ model (14) are integrated to produce the model output. The uncertainty of the model output and its discrepancy from the available data is quantified through a parametrized error model. The stochastic model and its quantified uncertainties are then used to identify the OpTS. The reader is referred to the Supplementary Materials of this paper for the definition of the utility function, the optimization algorithm as well as for the epidemiological and error models.

Beginning of the epidemic - Optimal testing without data At the start of an epidemic, there are no data and we assume no other prior information regarding the spread of the pathogen in a country. The initial conditions for the number of unreported infections $\left(\boldsymbol{I}_{\mathrm{IC}}^{u}\right)$ were selected with non-zero values for the cantons of Aargau, Bern, Basel-Landschaft, Basel-Stadt, Fribourg, Geneva, Grisons, St.Gallen, Ticino, Vaud, Valais and Zurich based on their population and their large number of interconnections. Due to the lack of any prior information and relevant data, 
all the parameters are assumed to follow uniform prior distributions (see table S5, for details).

In Switzerland the first infectious person was reported on February $25^{\text {th }}$ in the canton of Ticino $\left(I_{\mathrm{TI}}^{r}=1\right)$ with no initial reported infections in all other cantons. The initial number of exposed individuals is set proportional to the number of unreported infections $E_{k}=3 I_{k}^{u}$ in accordance with the value of $R_{0} \approx 3$ reported in (24) in the initial stage of the disease. The rest of the population is assumed to be susceptible. The methodology involves parameters of interest $(\boldsymbol{\vartheta}=(\beta, \mu, \alpha, Z, D, \theta, c))$ and nuisance parameters $\left(\widetilde{\boldsymbol{\vartheta}}=\left(\boldsymbol{I}_{\mathrm{IC}}^{u}, \tau\right)\right)$ that the testing strategy does not aim to determine (see Supplementary Material for definitions).

The estimated expected utility functions $\hat{U}(s)$ for up to four measurements in the cantons of Switzerland for a time horizon of 8 days is shown in Figure 2. Higher values for expected utility are estimated in cantons with larger population reflecting the larger relative uncertainty for cantons with only few reported cases. This implies that small cantons with little mobility rates are less preferred for performing tests since their contribution to the information gain is not significant. The Bayesian analysis enables the inference of the particular cantons and days for which tests should be performed in order to maximize the information gain. Accordingly, the most informative measurement should have been taken in Zurich on March the $2^{\text {nd }}$. Following the sequential optimization strategy, the optimal location and time for the second measurement is determined to be canton of Vaud on the $27^{\text {th }}$ of February. As expected, the information that is gained from tests in the canton of Vaud is less than the information gained from the canton of Zurich. The information that would have been gained by testing the next two preferred cantons of Vaud and Basel-Landschaft on March the $3^{\text {rd }}$ and February the $28^{\text {th }}$ respectively, is progressively reduced to a small level that, given the testing costs, does not justify carrying out tests in more than 4 cantons. The values of the optimal times are listed in table $\mathrm{S} 1$ in the Supplementary Materials.

The results indicate that the proposed OpTS selects certain populous and well intercon- 
medRxiv preprint doi: https://doi.org/10.1101/2020.07.20.20157818; this version posted July 27, 2020. The copyright holder for this preprint

nected cantons at specific times to acquire the most information for estimating the model parameters.

\section{Exponential spreading and optimal testing strategy during non-pharmaceutical interven-}

tions When the spreading of the coronavirus entered an exponential growth stage, several governments decided to take non-pharmaceutical interventions such as requesting social distancing, closing schools and restaurants, or even ordering a complete lockdown in order to contain the epidemic. Here, the goal of the OpTS is to obtain measurements that they help to better assess the effectiveness of these interventions.

In this case, priors for the model parameters are informed using data from the spread of the COVID-19. The daily reported infections in Switzerland (25) from the $25^{\text {th }}$ of February up to the $17^{\text {th }}$ of March 2020 are used to update the uniform prior distributions, specified in the previous phase, by using Bayesian inference (see figure S1 in Supplementary Materials). In the $S E I^{r} I^{u} R$ model we model the non-pharmaceutical interventions with a time-dependent infection rate $\beta$ and the mobility factor $\theta$. These parameters are also calibrated by the data and provide an estimate on the timing and effectiveness of the interventions (16).

Figure 3 shows the maximum values of the information gain for each measurement. For cantons with a small population and low connectivity to other cantons a low information gain is found. The opposite can be observed for cantons with large population and strong connections to other cantons. The values for the maximum utility in time for the measurements are listed in Table S2.

If only a single canton is to be selected, tests in the canton of Vaud carried out on the $30^{\text {th }}$ of March are to be preferred over tests in either of the cantons of Zurich, Bern or Geneva (blue in figure 3). If two tests can be afforded, the OpTS is to carry them out in the same canton (Vaud) on the $17^{\text {th }}$ and on the $30^{\text {th }}$ March (blue and green in figure 3). Note that the canton of 
medRxiv preprint doi: https://doi.org/10.1101/2020.07.20.20157818; this version posted July 27, 2020. The copyright holder for this preprint

Zurich, ranked as the next preferred canton for a single test (blue in figure 3), is not selected by the methodology since part of the information that would be gained from testing is already contained in tests performed in Vaud. The optimal location and time for a third test is the canton of Grisons on the $30^{\text {th }}$ of March (yellow in figure 3). The canton of Zurich is proposed as the fourth location to be tested on the $30^{\text {th }}$ of March as well. However, the information gain from the fourth test in the canton of Zurich is approximately $10 \%$ of the total information gained from the tests carried optimally in the first three cantons.

The results suggest that tests in two locations/times provide significant information regarding assessing the effectiveness of interventions. Further tests on more locations/times do not add substantial information. It is evident that a trade-off between the required information gain and cost of testing are decisive for the number of necessary tests.

Optimal monitoring for a second outbreak After the relaxation of measures that assisted in mitigating the initial spread of the disease, it is critical to monitor the population for a possible second outbreak (5). The proposed OpTS supports such monitoring with randomized tests of the population based on data up to and after the release of the measures.

First, Bayesian inference is performed with data available up to June the $6^{\text {th }}$, allowing for an update of the uniform priors. This date is in accordance to the first stage of major release of measures in Switzerland (26). The effects of interventions are modeled by a parametrized time-dependent infection rate and mobility factor (see Supplementary Materials for a detailed description). The inferred probability distributions of these additional parameters are taken into account as the OpTS maximizes the information gain.

Subsequently, data up to July the $9^{\text {th }}$ are included, repeating the Bayesian inference and estimating the OpTS (marginal distributions and predictions shown in Figures S3 and S4). The inference indicates that the relaxation of measures correlates with an increase in the number of 
medRxiv preprint doi: https://doi.org/10.1101/2020.07.20.20157818; this version posted July 27, 2020. The copyright holder for this preprint

reported infections as observed in Figure 4. The information gain for each canton indicates the most informative tests should be performed a week after performing the inference. The provided information could then assist in estimating the severity of a second outbreak as indicated by the maximum of the utility in time (Tables S3 and S4).

Given that tests should be carried out in four locations and times, the methodology promotes optimal tests for two different times, within a week, in the cantons of Zurich and Vaud. First, tests should be performed in Zurich, providing high information gain for both considered cases. The next two tests are to be performed in Zurich and Vaud, with a rank that depends on the considered case, while the fourth test should be performed in Vaud. We find that the information gain from the last test is approximately $10 \%$ of the cumulative information gain from the first three tests. The number of tests can be then selected according to the available resources.

Effectiveness of Optimal Testing We demonstrate the importance of following an OpTS by comparing it with a non-specific testing campaign. We first re-examine the situation at the start of an epidemic and assume that the available resources allow for two randomized tests. Tests are simulated by evaluating the epidemiological model with the maximum a-posteriori estimate (MPE) of the parameters obtained from the inference in phase II (exponential growth) of the epidemic. We used data of the first 21 days of the infection spread in Switzerland (25) (February $25^{\text {th }}$ to March $17^{\text {th }}$ ). After evaluating the model, artificial measurements are obtained by adding a stochastic error term.

For the optimal strategy, data are collected by consulting figure 2 . Thus, the two randomized tests are performed in the cantons of Zurich and Vaud, on the $2^{\text {nd }}$ of March and the $27^{\text {th }}$ of February respectively. For a non-specific strategy, the cantons of Ticino and Bern were selected, on the $28^{\text {th }}$ of February. These artificial data, obtained for the two strategies, are added to the real data of the daily reported cases from the first 8 days after the outbreak in Ticino. For the 
expanded data-set $\mathcal{D}$ the posterior distributions $p\left(\boldsymbol{\vartheta} \mid \widetilde{\vartheta}_{\mathrm{MPE}}, \mathcal{D}\right)$ are found by sampling the model parameters using nested sampling (17). Note that the value of $c$ is also inferred. For simplicity, the value of the correlation time $\tau$ is assumed to be known as this does not influence the results as long as the tests are carried out in different cantons.

The resulting one- and two-dimensional marginalized posterior distributions for both strategies are shown in figure 5. We note that the dispersion coefficient $r$ (defined in the Supplementary Materials) in the error model for the real data (the reported infections) and the correlation parameter are almost the same for both strategies. However the model parameters show significant differences even when only two new data-points are added to a set of 208 data-points.

The posterior distributions of the parameters of interest are propagated through the epidemiology model to provide the uncertainties in the number of unreported infectious individuals. In figure 6 the model output for the total number of unreported infections is plotted together with a $99 \%$ confidence interval along with the true value of the unreported cases obtained by using the selected parameters. The predictions from the OpTS have a much higher certainty with a confidence interval that is up to four times narrower than the one from a non-specific strategy. Further comparisons, demonstrating the value of the OpTS, include model predictions with higher certainty, as indicated by confidence intervals that are narrower than the ones obtained from from a non-specific strategy (figures S4 and S5, see Supplementary Material). Narrower uncertainty bounds provide higher confidence for decisions related to possible interventions to contain the epidemic.

\section{Discussion}

We introduced a systematic approach to identify optimal times and locations for randomized tests in order to quantify infectious individuals of a country's population during the COVID19 epidemic. The proposed OpTS exploits prior information and available data to maximize 
the expected information gain in parameters of interest and to minimize uncertainties in the forecasts of epidemiological models. In turn, improved forecasts for the virus spread provide rational guidelines for optimal allocation of finite testing resources.

The proposed method is demonstrated by focusing on the outbreak of the epidemic in Switzerland. The methodology relies on Bayesian experimental design using prior information and available data of reported infections along with forecasts from the $S E I^{r} I^{u} R$ model. We compute the optimal testing strategy for three phases of the epidemic. First, we quantify the spread of the disease after the first reported infection. At this stage the method identifies the most crucial dates and locations for randomized tests in the country's population. The deployment of OpTS at this phase would have allowed authorities to perform randomized testing in a period of high uncertainty, well in advance of the disease outbreak. During the period of interventions the proposed strategy would help quantify their effectiveness assisting decision making for further interventions or retraction of measures that may be harmful to the economy. Finally, the OpTS can assist monitoring for a recurrence of the disease after preventive measures have been relaxed and help guide further planing of interventions.

We remark that the proposed OpTS does not depend on a particular type of data/model or to the country of Switzerland. The open source code is modular, scalable and readily adaptable to different scenarios for the epidemic and countries around the world. We believe that the present work can be a valuable tool for decision makers to allocate resources efficiently for testing the population, providing a reliable quantification of the spread of the disease and designing effective interventions.

\section{References}

1. D. Adam, Special report: The simulations driving the world's response to COVID-19. $\mathrm{Na}$ ture 580, 316 (2020). 
medRxiv preprint doi: https://doi.org/10.1101/2020.07.20.20157818; this version posted July 27, 2020. The copyright holder for this preprint (which was not certified by peer review) is the author/funder, who has granted medRxiv a license to display the preprint in perpetuity.

All rights reserved. No reuse allowed without permission.

2. N. Zhu et al., A Novel Coronavirus from Patients with Pneumonia in China, 2019. New England Journal of Medicine 382, 727 (2020).

3. A. Du Toit, Outbreak of a novel coronavirus. Nature Reviews Microbiology 18, 123 (2020).

4. J. Ioannidis, The infection fatality rate of COVID-19 inferred from seroprevalence data. medRxiv (2020).

5. J. Haushofer C. J. E. Metcalf, Which interventions work best in a pandemic? Science 368, 1063 (2020).

6. M. Esbin et al., Overcoming the bottleneck to widespread testing: A rapid review of nucleic acid testing approaches for COVID-19 detection. RNA 26, rna.076232.120 (2020).

7. J. Eberhardt, N. Breuckmann C. Eberhardt, Multi-Stage Group Testing Improves Efficiency of Large-Scale COVID-19 Screening. Journal of Clinical Virology 128, 104382 (2020).

8. N. Sinnott-Armstrong, D. Klein B. Hickey, Evaluation of Group Testing for SARS-CoV-2 RNA. medRxiv (2020).

9. J. Dehning et al., Inferring change points in the spread of COVID-19 reveals the effectiveness of interventions. Science (2020).

10. K. Chaloner I. Verdinelli, Bayesian Experimental Design: A Review. Statistical Science 10, 273 (1995).

11. X. Huan Y. M. Marzouk, Simulation-based optimal Bayesian experimental design for nonlinear systems. Journal of Computational Physics 232, 288 (2013).

12. E. G. Ryan, C. C. Drovandi, J. M. McGree A. N. Pettitt, A Review of Modern Computational Algorithms for Bayesian Optimal Design. International Statistical Review 84, 128 (2016). 
medRxiv preprint doi: https://doi.org/10.1101/2020.07.20.20157818; this version posted July 27, 2020. The copyright holder for this preprint (which was not certified by peer review) is the author/funder, who has granted medRxiv a license to display the preprint in perpetuity.

All rights reserved. No reuse allowed without permission.

13. A. E. Gongora et al., A Bayesian experimental autonomous researcher for mechanical design. Science Advances 6 (2020).

14. R. Li et al., Substantial undocumented infection facilitates the rapid dissemination of novel coronavirus (SARS-CoV-2). Science 368, 489 (2020).

15. E. T. Jaynes, Probability Theory: The Logic of Science (Cambridge University Press, 2003).

16. P. Karnakov et al., Data driven inference of the reproduction number for COVID-19 before and after interventions for 51 European countries. Swiss Medical Weekly (2020).

17. J. S. Speagle, Dynesty: a dynamic nested sampling package for estimating Bayesian posteriors and evidences. Monthly Notices of the Royal Astronomical Society 493, 3132-3158 (2020).

18. D. Lindley, On a measure of the information provided by an experiment. Annals of mathematical statistics 27, 986 (1956).

19. E. Karni D. Schmeidler (Elsevier, 1991), vol. 4 of Handbook of Mathematical Economics, pp. $1763-1831$.

20. K. J. Ryan, Estimating Expected Information Gains for Experimental Designs With Application to the Random Fatigue-Limit Model. Journal of Computational and Graphical Statistics 12, 585 (2003).

21. P. Tsilifis, R. G. Ghanem P. Hajali, Efficient Bayesian Experimentation Using an Expected Information Gain Lower Bound. SIAM/ASA Journal on Uncertainty Quantification 5, 30 (2017).

22. J. Tolles T. Luong, Modeling Epidemics With Compartmental Models. JAMA 323, 2515 (2020). 
medRxiv preprint doi: https://doi.org/10.1101/2020.07.20.20157818; this version posted July 27, 2020. The copyright holder for this preprint (which was not certified by peer review) is the author/funder, who has granted medRxiv a license to display the preprint in perpetuity.

All rights reserved. No reuse allowed without permission.

23. C. Papadimitriou, Optimal sensor placement methodology for parametric identification of structural systems. Journal of Sound and Vibration 278, 923 (2004).

24. Y. Liu, A. A. Gayle, A. Wilder-Smith J. Rocklöv, The reproductive number of COVID-19 is higher compared to SARS coronavirus. Journal of Travel Medicine 27 (2020).

25. Statistisches Amt Kanton Zürich, SARS-CoV-2 Cases communicated by Swiss Cantons and Principality of Liechtenstein (FL), https://github.com/openZH/covid_19 (2020). Online.

26. Bundesamt für Gesundheit, Neues Coronavirus, https://www.bag.admin.ch/bag/de/home/ krankheiten/ausbrueche-epidemien-pandemien/aktuelle-ausbrueche-epidemien/novelcov.html (2020). Online.

\section{Acknowledgments}

We acknowledge discussions with Fabian Wermelinger, Lucas Amoudruz, Martin Boden (ETHZ). Sergio Martin (ETHZ) provided technical assistance with the software. Funding: We acknowledge funding by ETH Zurich and computing resources by the Swiss Supercomputing center (CSCS). Authors contributions: Conceptualization: C.P., P.Ko.; Data curation: M.C., P.W.; Formal Analysis: M.C., P.W., G.A., D.W., C.P.; Funding acquisition: P.Ko.; Investigation: M.C., P.W., C.P., G.A., P.Ko. Methodology: P.W., M.C., C.P., G.A., P.Ko.; Project administration: P.Ko.; Resources: P.Ko.; Software: P.W., M.C., D.W., I.K., P.Ka.; Supervision: C.P., P.Ko., G.A.; Validation: M.C., P.W.; Visualization: P.W., M.C., P.Ka; Writing - original draft: P.W., M.C., C.P., P.Ko.; Writing - review \& editing: P.W., M.C., C.P., P.Ko., G.A., D.W., I.K., P.Ka. Competing interests: The authors have no competing interests. Data and materials availability: All data is available in the manuscript or the Supplementary Materials. 
medRxiv preprint doi: https://doi.org/10.1101/2020.07.20.20157818; this version posted July 27, 2020. The copyright holder for this preprint (which was not certified by peer review) is the author/funder, who has granted medRxiv a license to display the preprint in perpetuity.

All rights reserved. No reuse allowed without permission.

322

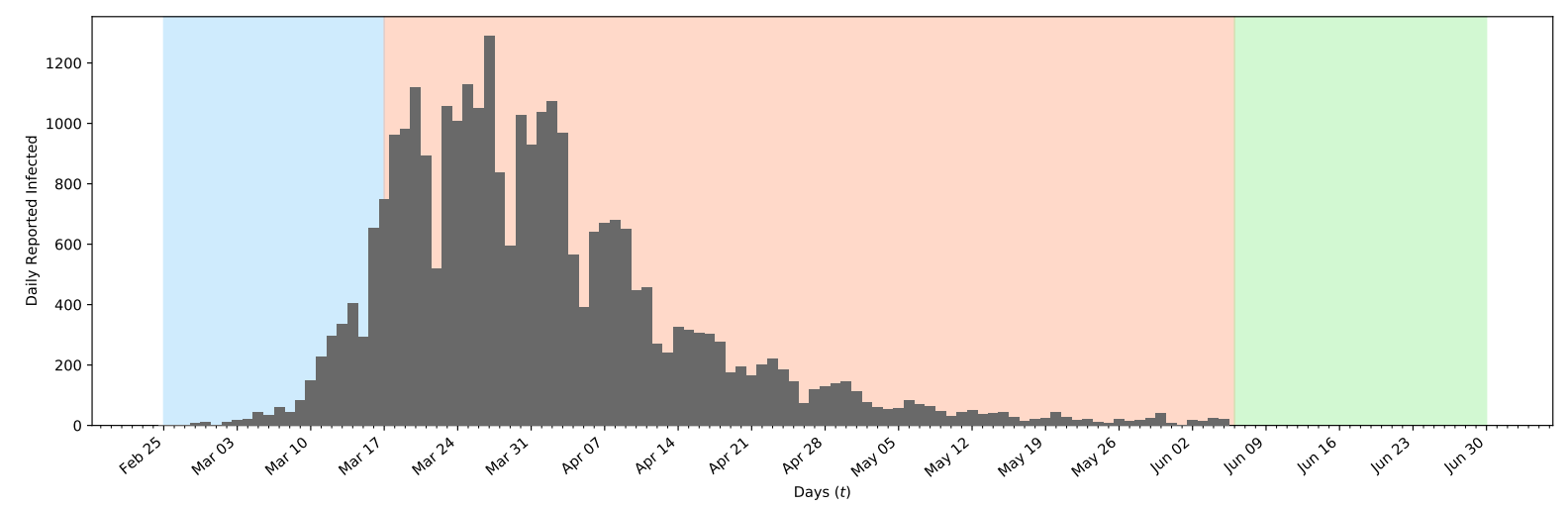

Figure 1: Testing scenarios for the COVID19 outbreak in Switzerland. Daily reported Coronavirus cases in Switzerland are plotted as gray bars. The period before (blue), during (red) and after (green) imposing non-pharmaceutical interventions are marked with color. 

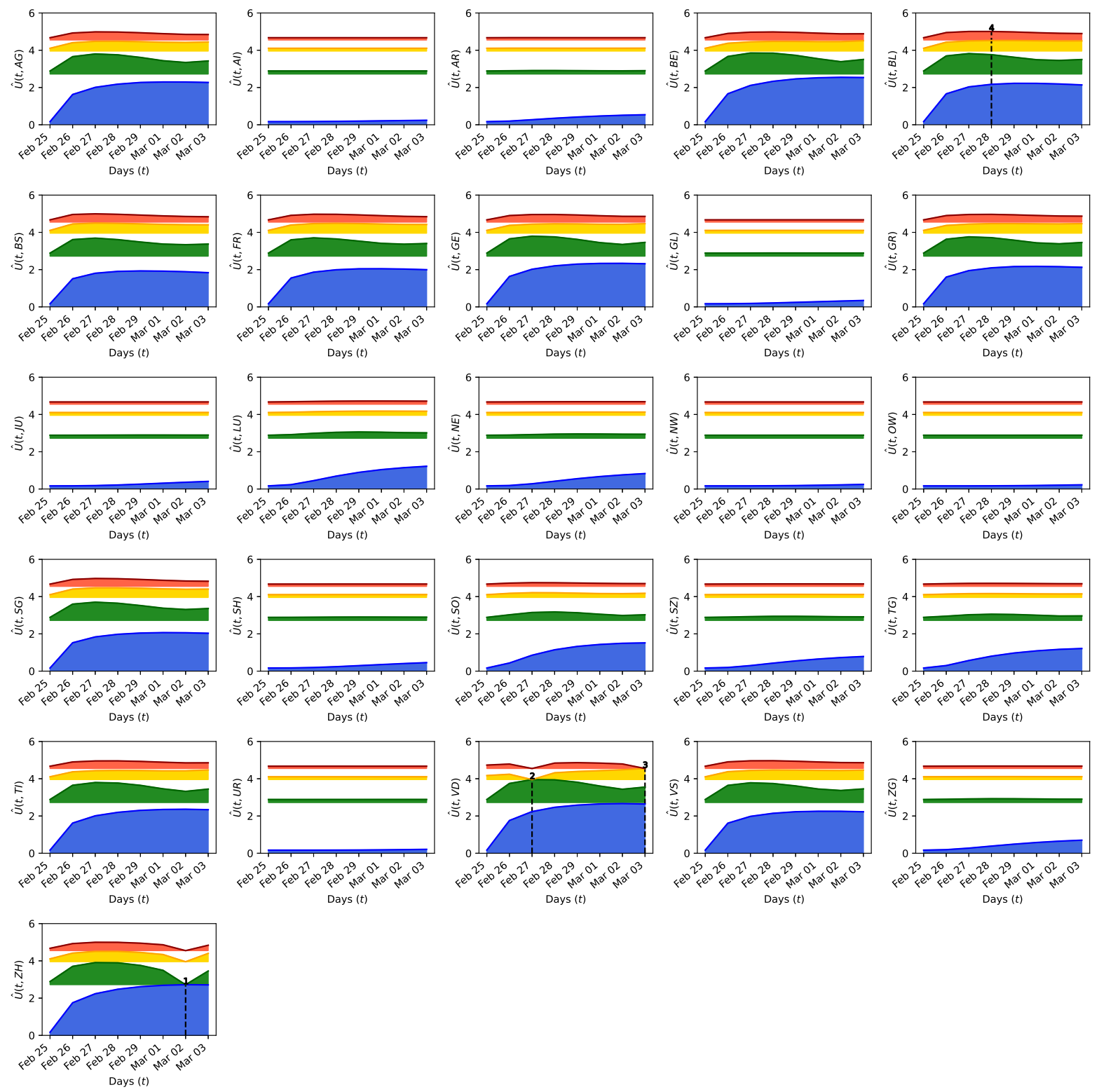

Figure 2: Expected information gain during start of epidemic. The blue curve corresponds to the utility of taking one measurement. The green curve is the utility when a second measurement is added, provided the location and time of the first measurement correspond to the maximum of the blue curve (found in the canton of Zurich, on March $2^{\text {nd }}$ ). Similarly, the yellow and red curves show the utilities for a third and fourth measurements, when the locations and time of the previous measurements are fixed to their optimal values. The fixed dates and location of each measurement are plotted with black dashed lines. The shaded areas indicate the difference to the expected information gain of the previous measurement, which becomes thinner as additional measurements do not yield a further significant information gain. 

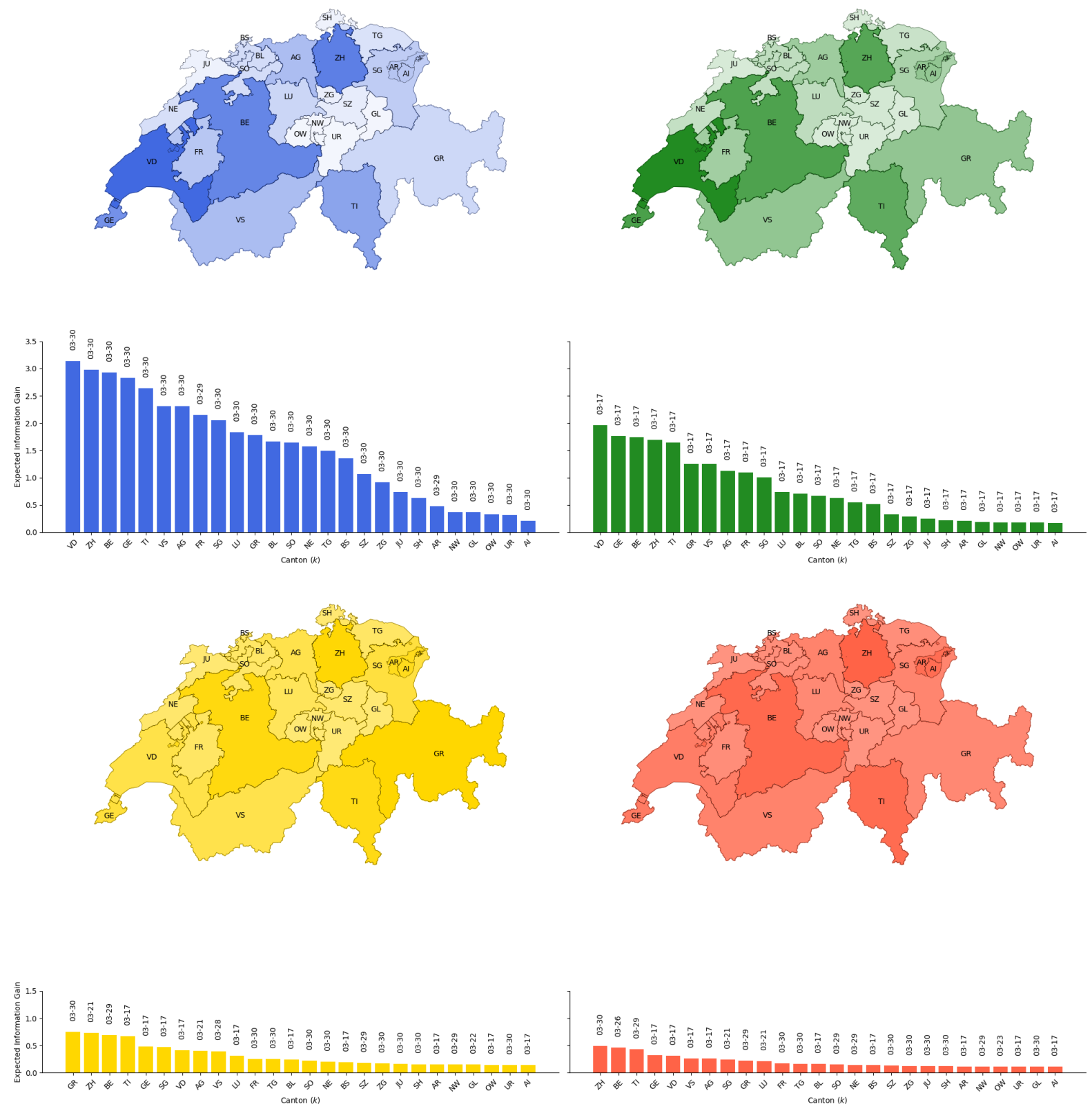

Figure 3: Optimal testing strategy for effect of non-pharmaceutical interventions. The maximum gain of information is plotted on the map of Switzerland. Here blue corresponds to taking one measurement, green to adding a second, yellow to a third and red to a fourth. Below the map we plot the magnitude of the expected information gain of each measurement, along with the optimal measurement dates per canton. 


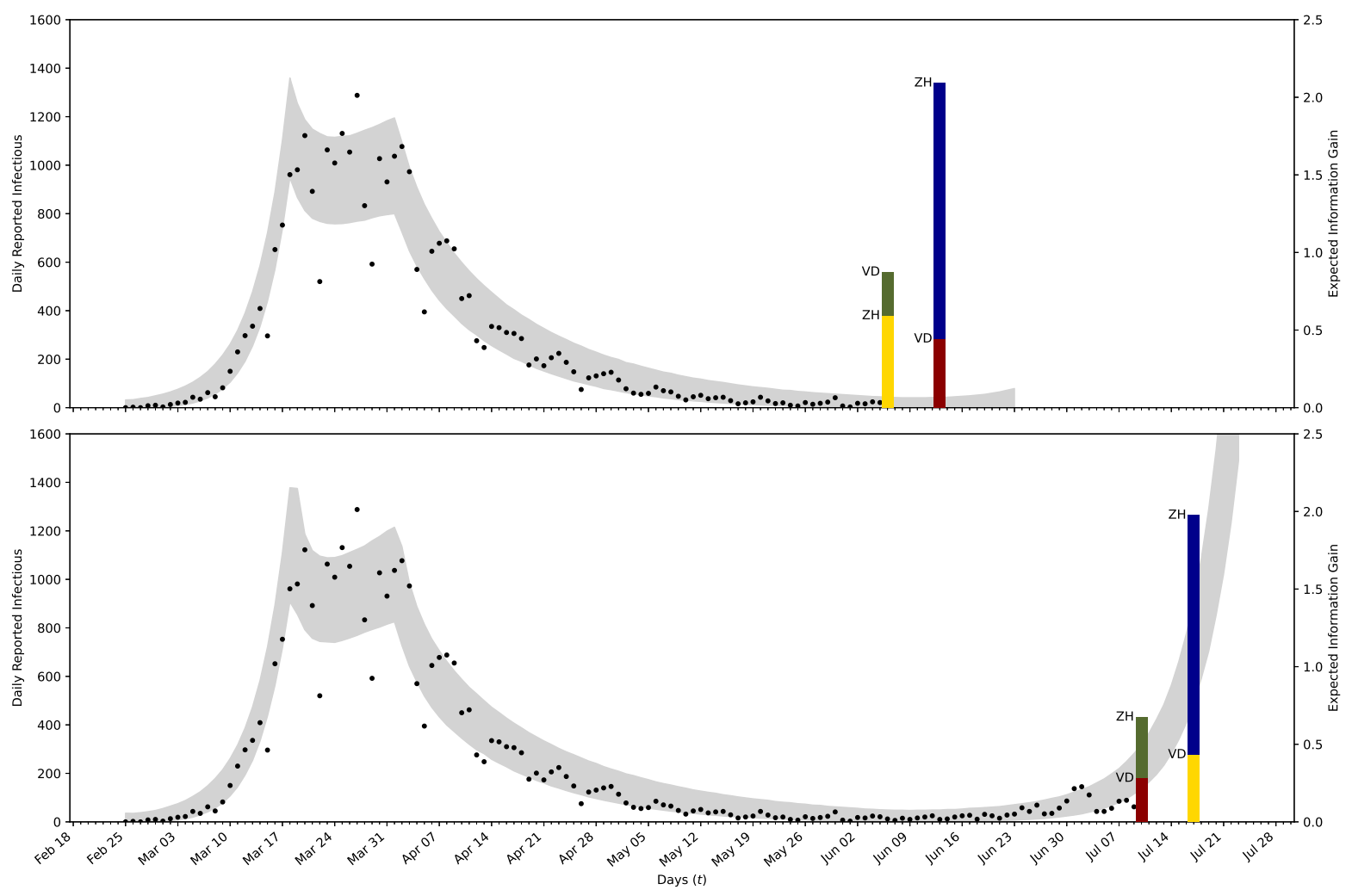

Figure 4: Optimal testing strategy to monitor a second outbreak. We use Bayesian inference to determine the parameters of the first infection wave using the data (black dots) of the daily new reported infections up to the $6^{\text {th }}$ of June (upper plot) and to the $9^{\text {th }}$ of July (lower plot). The $99 \%$ confidence intervals are plotted in gray. The proposed testing strategy is plotted with vertical bars at the found optimal days. Here blue indicated the utilities for the first measurement. The green bars correspond to the gain in utility when adding a second measurement assuming the first was chosen in the optimal location, where the yellow and red correspond to adding a third and fourth measurement. 

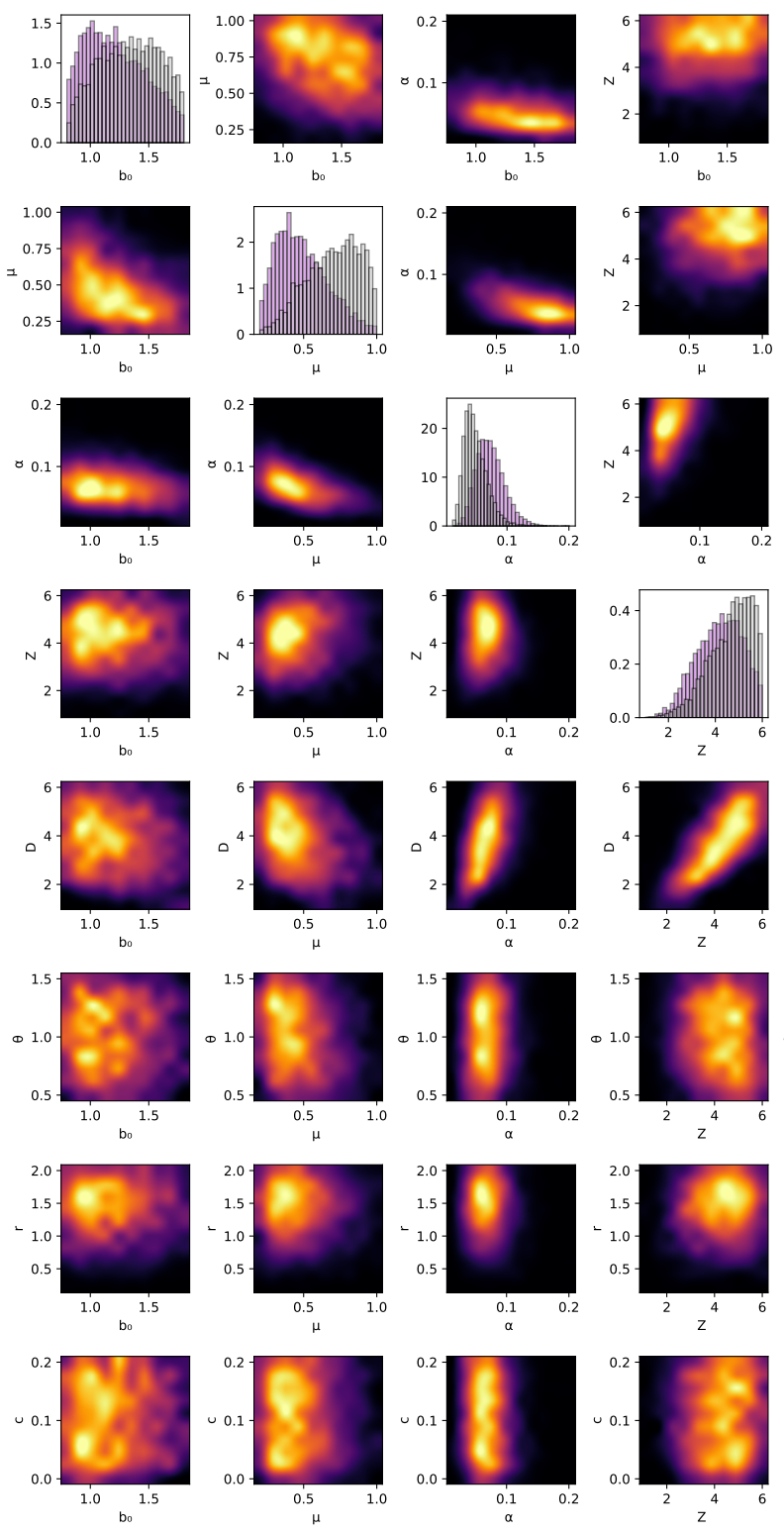

0.5
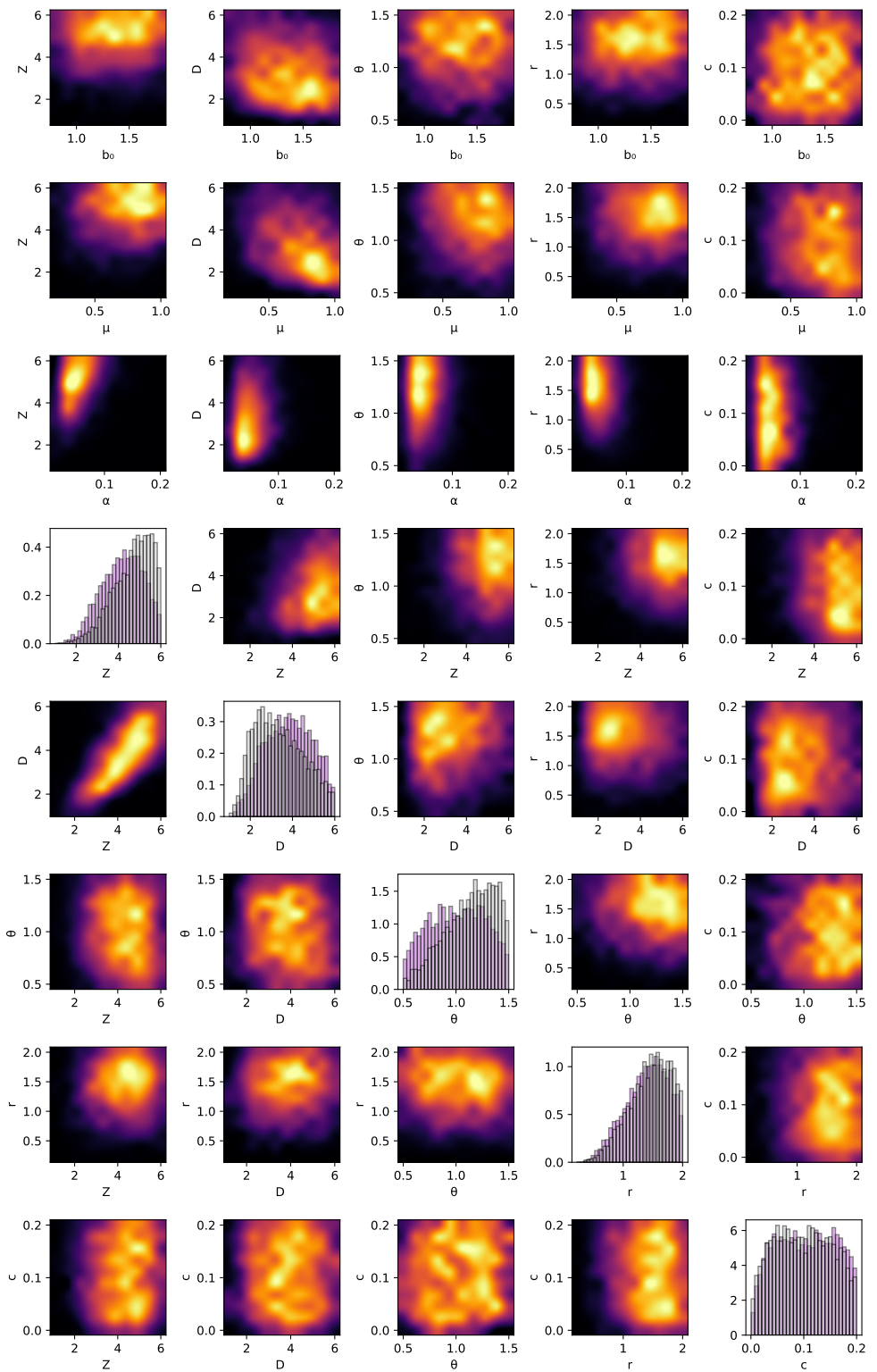

Figure 5: Marginal posterior distributions for two strategies. The diagonal shows the histogram for the marginal distribution for every parameter. Purple indicates posterior for the the measurement following the optimal testing strategy, gray the one for the non-specific strategy. The lower half and upper half show the samples of the joint distribution of two parameters for the optimal and the non-specific strategy respectively. Here black indicates low density and yellow high density. 


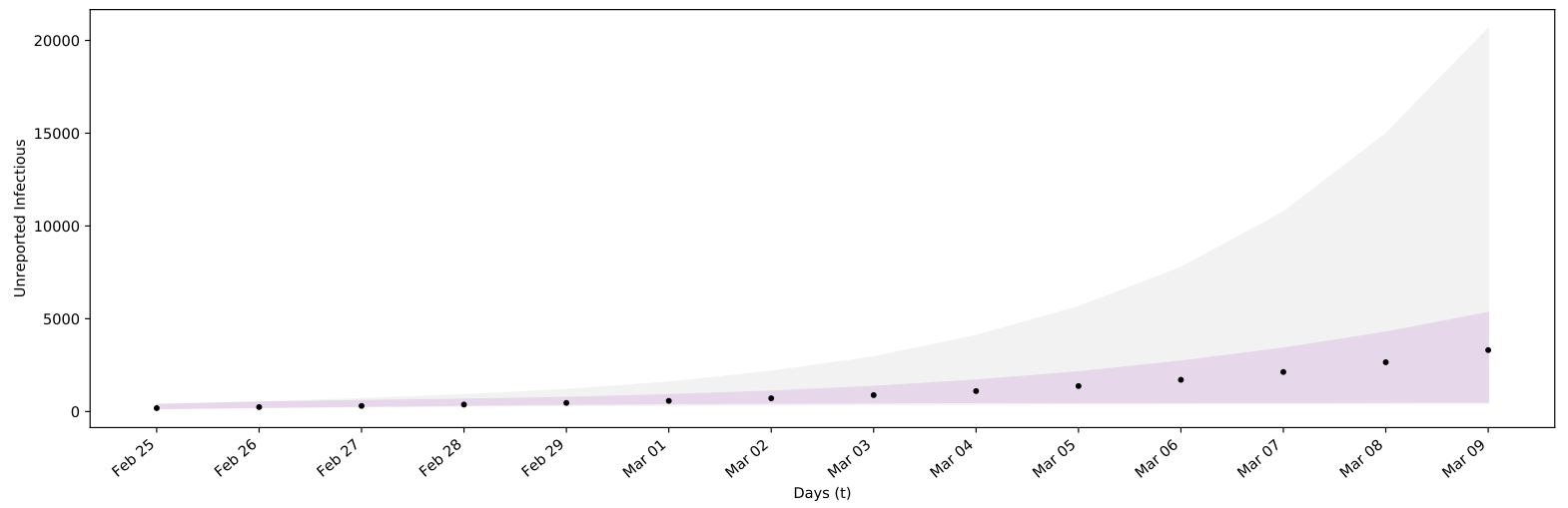

Figure 6: Prediction uncertainty for different testing strategies. The black dots show the actual unreported infectious for an artificial spread in Switzerland. The error bounds show the $99 \%$ confidence intervals of the model output for samples of the parameters with data obtained by optimal (purple) and non-specific testing (gray). 


\title{
Supplementary Materials for
}

\section{Optimal Testing Strategy for the Identification of COVID-19 Infections}

\author{
Michail Chatzimanolakis ${ }^{1, *}$, Pascal Weber ${ }^{1, *}$, George Arampatzis ${ }^{1}$, \\ Daniel Wälchli ${ }^{1}$, Ivica Kičić ${ }^{1}$, Petr Karnakov ${ }^{1}$, Costas Papadimitriou ${ }^{2}$, \\ Petros Koumoutsakos ${ }^{1, \dagger}$ \\ ${ }^{1}$ Computational Science and Engineering Laboratory, ETH Zurich, \\ Clausiusstrasse 33, CH-8092, Switzerland \\ ${ }^{2}$ Department of Mechanical Engineering, University of Thessaly, \\ Pedion Areos, GR-38334 Volos, Greece \\ * These authors contributed equally to this work. \\ † Corresponding author. E-mail: petros@ethz.ch
}

\section{This PDF file includes:}

Materials and Methods

Fig S1 - S6

Table S1 - S5

References 


\section{Materials and Methods}

The optimal time (day) and location (canton) for testing a population to detect infectious individuals is determined via Bayesian optimal experimental design $(12,13,27)$. This optimal testing strategy (OpTS) relies on combining Bayesian inference and utility theory with forecasting models of the epidemic. We remark that the OpTS does not depend on a particular epidemiological model or type of data. The methodology is applicable at all stages of the epidemic (inception to re-occurrence). It can operate without data at the early stages of the pandemic and takes advantage of data available at later stages of the pandemic. The methodology is rendered computationally efficient using a sequential optimization algorithm (23).

Bayesian Inference from randomized testing We consider a testing campaign including a set $(s)$ of randomized tests $s_{i}=\left(k_{i}, t_{i}\right), i=1, \ldots M_{y}$ performed in location $k_{i}$ and on day $t_{i}$. These tests measure a quantity of interest (QoI), that is denoted by $\boldsymbol{y}(\boldsymbol{s})=\left(y_{1}, \ldots, y_{M_{y}}\right)$. Here, $y_{i}$ is the number of unreported infectious individuals, measured through test $s_{i}$. The QoI can be predicted by a model $\boldsymbol{g}(\boldsymbol{s}, \boldsymbol{\vartheta}, \widetilde{\boldsymbol{\vartheta}})$ (here the $S E I^{r} I^{u} R$ epidemiological model) that depends on parameters of interest $\vartheta \in \mathbb{R}^{N}$ and nuisance parameters $\widetilde{\boldsymbol{\vartheta}} \in \mathbb{R}^{\tilde{N}}$. The distinction between model and nuisance parameters is discussed in later sections. We note that both sets of parameters are uncertain and the proposed method aims to reduce the uncertainty only in the parameters of interest.

A stochastic error term $\varepsilon(s)$ links the model prediction with the QoI :

$$
\boldsymbol{y}(\boldsymbol{s})=\boldsymbol{g}(\boldsymbol{s}, \boldsymbol{\vartheta}, \widetilde{\boldsymbol{\vartheta}})+\boldsymbol{\varepsilon}(\boldsymbol{s})
$$

The error $\varepsilon(s)$ is assumed to follow a zero-mean multivariate normal distribution $\mathcal{N}(0, \Sigma)$ with covariance matrix $\Sigma \in \mathbb{R}^{M_{y} \times M_{y}}$. The elements of the covariance matrix $\left(\Sigma_{s, s^{\prime}}\right)$ correspond to 
measurements taken at $s=(k, t)$ and $s^{\prime}=\left(k^{\prime}, t^{\prime}\right)$ and are given by

$$
\Sigma_{s, s^{\prime}}=\sigma_{t} \sigma_{t^{\prime}} \exp \left(-\frac{\left|t-t^{\prime}\right|}{\tau}\right) \delta_{k k^{\prime}}
$$

where $\delta_{k k^{\prime}}$ is the Kronecker delta, which is 1 for $k=k^{\prime}$ and 0 otherwise. The correlation time $\tau \in[0.5,3.5]$ is considered a nuisance parameter. These assumptions about the covariance imply that measurements in different locations are not correlated, while those in the same location have an exponentially decaying temporal correlation. The latter avoids clustering of measurements in small time intervals $(28,29)$. The factor $\sigma_{t} \in \mathbb{R}$ is assumed proportional to the expectation of the QoI, taken over all possible test locations and over the range of model and nuisance parameters

$$
\sigma_{t}=c \frac{1}{K} \sum_{i=1}^{K} \mathbb{E}_{\boldsymbol{\vartheta}, \widetilde{\vartheta}}\left[g\left(s_{i}, \boldsymbol{\vartheta}, \widetilde{\boldsymbol{\vartheta}}\right)\right],
$$

where $s_{i}=(i, t)$. The parameter $c \in[0,0.25]$ is considered a model parameter. The expectation $\mathbb{E}_{\vartheta, \widetilde{\vartheta}}[\cdot]$ is taken with respect to all parameters $\vartheta$ and $\widetilde{\vartheta}$ that follow the prior probability distribution with density $p(\boldsymbol{\vartheta}, \widetilde{\boldsymbol{\vartheta}})=p(\boldsymbol{\vartheta}) p(\widetilde{\boldsymbol{\vartheta}})$.

Under these assumptions, the conditional probability of $\boldsymbol{y}$ on $\boldsymbol{\vartheta}, \widetilde{\vartheta}$ and $\boldsymbol{s}$ is given by

$$
\begin{aligned}
& p(\boldsymbol{y} \mid \boldsymbol{\vartheta}, \widetilde{\boldsymbol{\vartheta}}, \boldsymbol{s})= \\
& \quad \frac{1}{\sqrt{(2 \pi)^{M_{y}|\Sigma(\boldsymbol{s})|}}} \exp \left(-\frac{1}{2}(\boldsymbol{y}(\boldsymbol{s})-\boldsymbol{g}(\boldsymbol{s}, \boldsymbol{\vartheta}, \widetilde{\boldsymbol{\vartheta}}))^{\top} \Sigma(\boldsymbol{s})^{-1}(\boldsymbol{y}(\boldsymbol{s})-\boldsymbol{g}(\boldsymbol{s}, \boldsymbol{\vartheta}, \widetilde{\boldsymbol{\vartheta}}))\right),
\end{aligned}
$$

where $|\Sigma(s)|$ is the determinant of the covariance matrix.

\section{Expected Information Gain The most informative measurements $\boldsymbol{y}$ provide the least un-} certainty in the estimates of the model parameters $\vartheta$. Starting with a user-postulated prior distribution $p(\boldsymbol{\vartheta})$, Bayesian learning is used to update the uncertainties in the model parameters leading to a posterior distribution $p(\boldsymbol{\vartheta} \mid \boldsymbol{y}, \widetilde{\vartheta}, s)$, based on the information contained in the test data $\boldsymbol{y}$. The Kullback-Leibler (KL) divergence between the posterior $p(\boldsymbol{\vartheta} \mid \boldsymbol{y}, \widetilde{\boldsymbol{\vartheta}}, \boldsymbol{s})$ and the prior distributions $p(\boldsymbol{\vartheta})$ of the model parameters measures the distance between the two distributions. 
Informative data produce posterior distributions that differ from the prior; greater differences lead to higher information gain. Therefore, the most informative data $\boldsymbol{y}$ correspond to the testing strategy (measurement locations and times) with the highest information gain $(18,19)$.

The OpTS is identified by maximizing a utility function $(12,20)$. One choice is the KL divergence $u(\boldsymbol{y}, \widetilde{\boldsymbol{\vartheta}}, \boldsymbol{s})=D_{\mathrm{KL}}(p(\boldsymbol{\vartheta} \mid \boldsymbol{y}, \widetilde{\boldsymbol{\vartheta}}, \boldsymbol{s}) \| p(\boldsymbol{\vartheta}))$ quantifying the information gain from the data (12). However, since data are not available in the experimental design phase, the utility function is selected here to be the expected $\mathrm{KL}$ divergence $\mathbb{E}_{\boldsymbol{y} \mid \widetilde{\boldsymbol{\vartheta}}, s}[u(\boldsymbol{y}, \widetilde{\boldsymbol{\vartheta}}, \boldsymbol{s})]$ over all data generated by the model prediction error equation (1). Also, to account for the uncertainty in nuisance parameters $\widetilde{\boldsymbol{\vartheta}}$, encoded in the prior distribution $p(\widetilde{\boldsymbol{\vartheta}})$, the expectation is also taken with respect to $\widetilde{\vartheta}$, which results in the utility function $(20)$

$$
\begin{aligned}
U(\boldsymbol{s}) & =\mathbb{E}_{\widetilde{\boldsymbol{\vartheta}}}\left[\mathbb{E}_{\boldsymbol{y} \mid \widetilde{\boldsymbol{\vartheta}}, \boldsymbol{s}}[u(\boldsymbol{y}, \widetilde{\boldsymbol{\vartheta}}, \boldsymbol{s})]\right] \\
& =\iiint \log \left(\frac{p(\boldsymbol{\vartheta} \mid \boldsymbol{y}, \widetilde{\boldsymbol{\vartheta}}, \boldsymbol{s})}{p(\boldsymbol{\vartheta})}\right) p(\boldsymbol{\vartheta} \mid \boldsymbol{y}, \widetilde{\boldsymbol{\vartheta}}, \boldsymbol{s}) \mathrm{d} \boldsymbol{\vartheta} p(\boldsymbol{y} \mid \widetilde{\boldsymbol{\vartheta}}, \boldsymbol{s}) \mathrm{d} \boldsymbol{y} p(\widetilde{\boldsymbol{\vartheta}}) \mathrm{d} \widetilde{\boldsymbol{\vartheta}}
\end{aligned}
$$

By using Bayes' theorem

$$
p(\boldsymbol{\vartheta} \mid \boldsymbol{y}, \widetilde{\boldsymbol{\vartheta}}, \boldsymbol{s})=\frac{p(\boldsymbol{y} \mid \boldsymbol{\vartheta}, \widetilde{\boldsymbol{\vartheta}}, \boldsymbol{s}) p(\boldsymbol{\vartheta})}{p(\boldsymbol{y} \mid \widetilde{\boldsymbol{\vartheta}}, \boldsymbol{s})}
$$

the utility function can be simplified to

$$
U(\boldsymbol{s})=\iiint \log \left(\frac{p(\boldsymbol{y} \mid \boldsymbol{\vartheta}, \widetilde{\boldsymbol{\vartheta}}, \boldsymbol{s})}{p(\boldsymbol{y} \mid \widetilde{\boldsymbol{\vartheta}}, \boldsymbol{s})}\right) p(\boldsymbol{y} \mid \boldsymbol{\vartheta}, \widetilde{\boldsymbol{\vartheta}}, \boldsymbol{s}) p(\boldsymbol{\vartheta}) p(\widetilde{\boldsymbol{\vartheta}}) \mathrm{d} \boldsymbol{y} \mathrm{d} \boldsymbol{\vartheta} \mathrm{d} \widetilde{\boldsymbol{\vartheta}}
$$

Note that the expected utility only depends on the locations and times of the measurements via $\boldsymbol{s}$. The term $p(\boldsymbol{y} \mid \widetilde{\boldsymbol{\vartheta}}, \boldsymbol{s})$ is the model evidence given by

$$
p(\boldsymbol{y} \mid \widetilde{\boldsymbol{\vartheta}}, \boldsymbol{s})=\int p(\boldsymbol{y} \mid \boldsymbol{\vartheta}, \widetilde{\boldsymbol{\vartheta}}, \boldsymbol{s}) p(\boldsymbol{\vartheta}) \mathrm{d} \boldsymbol{\vartheta}
$$

The choice of the prior distribution $p(\boldsymbol{\vartheta})$ for the parameters allows to incorporate prior knowledge from epidemiology. If no information is available from data, a case encountered in the 
beginning of the infection, a uniform prior distribution can be assumed. Table S5 summarizes our choice of prior distributions for all the involved uncertain quantities. If data $\boldsymbol{d}$ of the daily number of reported infectious individuals is available, Bayesian inference can be used to inform the prior distribution, as described later on. In this case, the prior $p(\vartheta)$ in equation (7) is replaced by the distribution $p(\boldsymbol{\vartheta} \mid \boldsymbol{d})$ informed from the data $\boldsymbol{d}$.

In the present work, the assumed nuisance parameters are the correlation time $\tau$ and the initial condition of the unreported infections in the cantons of Aargau, Bern, Basel-Landschaft, Basel-Stadt, Fribourg, Geneva, Grisons, St.Gallen, Ticino, Vaud, Valais and Zurich $\boldsymbol{I}_{\mathrm{IC}}^{u}=$ $\left(I_{\mathrm{AR}}^{u}, I_{\mathrm{BE}}^{u}, I_{\mathrm{BL}}^{u}, I_{\mathrm{BS}}^{u}, I_{\mathrm{FR}}^{u}, I_{\mathrm{GE}}^{u}, I_{\mathrm{GR}}^{u}, I_{\mathrm{SG}}^{u}, I_{\mathrm{TI}}^{u}, I_{\mathrm{VD}}^{u}, I_{\mathrm{VS}}^{u}, I_{\mathrm{ZH}}^{u}\right)$, with prior distributions $\boldsymbol{I}_{\mathrm{IC}}^{u} \sim \mathcal{U}\left([0,50]^{12}\right)$ and $\tau \sim \mathcal{U}([0.5,3.5])$.

Epidemiological Model Here we employ the $S E I^{r} I^{u} R$ epidemiological model (14) to forecast the dynamics of the coronavirus outbreak in Switzerland

$$
\begin{aligned}
\frac{\mathrm{d} S_{k}}{\mathrm{~d} t} & =-\frac{\beta S_{k} I_{k}^{r}}{N_{k}}-\frac{\mu \beta S_{k} I_{k}^{u}}{N_{k}}+\theta \sum_{l=1}^{K} \frac{M_{k l} S_{l}}{N_{l}-I_{l}^{r}}-\theta \sum_{l=1}^{K} \frac{M_{l k} S_{k}}{N_{k}-I_{k}^{r}} \\
\frac{\mathrm{d} E_{k}}{\mathrm{~d} t} & =\frac{\beta S_{k} I_{k}^{r}}{N_{k}}+\frac{\mu \beta S_{k} I_{k}^{u}}{N_{k}}-\frac{E_{k}}{Z}+\theta \sum_{l=1}^{K} \frac{M_{k l} E_{l}}{N_{l}-I_{l}^{r}}-\theta \sum_{l=1}^{K} \frac{M_{l k} E_{k}}{N_{k}-I_{k}^{r}} \\
\frac{\mathrm{d} I_{k}^{r}}{\mathrm{~d} t} & =\alpha \frac{E_{k}}{Z}-\frac{I_{k}^{r}}{D} \\
\frac{\mathrm{d} I_{k}^{u}}{\mathrm{~d} t} & =(1-\alpha) \frac{E_{k}}{Z}-\frac{I_{k}^{u}}{D}+\theta \sum_{l=1}^{K} \frac{M_{k l} I_{l}^{u}}{N_{l}-I_{l}^{r}}-\theta \sum_{l=1}^{K} \frac{M_{l k} I_{k}^{u}}{N_{k}-I_{k}^{r}} \\
\frac{\mathrm{d} N_{k}}{\mathrm{~d} t} & =\theta \sum_{l=1}^{K}\left(M_{k l}-M_{l k}\right),
\end{aligned}
$$

where $S_{k}, E_{k}, I_{k}^{r}$ and $I_{k}^{u}$ denote the number of individuals in canton $k=\{1, \ldots, K\}$ that are susceptible, exposed, reported infectious and unreported infectious, respectively. We denote by $K$ the number of cantons (26 in Switzerland), by $N_{k}$ the total population of the canton $k$, while the population mobility between cantons $k$ and $l$ is denoted by $M_{k l}$ with values obtained from 
the Swiss Federal Statistical Office (30). The model parameters are the transmission rate $(\beta)$, the relative transmission rate between reported and unreported infectious individuals $(\mu)$, the virus latency period $(Z)$, the infectious period $(D)$, the reporting rate $(\alpha)$ and the mobility factor $(\theta)$

We employ different time-dependent expressions for the transmission rate and the mobility factor for each stage of the epidemic. Constants are chosen for the start of an epidemic while in the cases of monitoring of interventions, the following expressions are used:

$$
\beta(t)=\left\{\begin{array}{ll}
b_{0}, & 0 \leq t \leq \delta_{1} \\
b_{1}, & \delta_{1}<t
\end{array}, \quad \theta(t)= \begin{cases}\theta_{0}, & 0 \leq t \leq \delta_{1} \\
\theta_{1}, & \delta_{1}<t\end{cases}\right.
$$

where $b_{0}, b_{1}, \theta_{0}$ and $\theta_{1}$ are the infection rates and mobility factors before and after the intervention. Time $t=0$ corresponds to the $25^{\text {th }}$ of February 2020, and $\delta_{1}=21$ to the $17^{\text {th }}$ of March 2020, when the lockdown was announced in Switzerland (26). Finally, for the third case (monitoring of a second outbreak) we assume that

$$
\beta(t)=\left\{\begin{array}{ll}
b_{0}, & 0 \leq t \leq \delta_{1} \\
b_{1}, & \delta_{1}<t \leq \delta_{2} \\
b_{2}, & \delta_{2}<t \leq \delta_{3} \\
b_{3}(t), & \delta_{3}<t
\end{array}, \quad \theta(t)= \begin{cases}\theta_{0}, & 0 \leq t \leq \delta_{1} \\
\theta_{1}, & \delta_{1}<t \leq \delta_{2} \\
\theta_{2}, & \delta_{2}<t \leq \delta_{3} \\
\theta_{0}, & \delta_{3}<t\end{cases}\right.
$$

As in equation (10), $b_{0}$ is the infection rate before the intervention while $b_{1}=c_{1} b_{0}$ and $b_{2}=$ $c_{2} b_{0}$ with $c_{1}, c_{2} \in[0,1]$ are the infection rates after the two interventions. Similarly, $\theta_{0}$ is the mobility factor before any interventions took place, while $\theta_{1}=c_{3} \theta_{0}$ and $\theta_{2}=c_{4} \theta_{0}$ with $c_{3}, c_{4} \in$ $[0,1]$ are the mobility factors after the two interventions. Moreover, $\delta_{1}$ and $\delta_{2}$ correspond to the days of the interventions. The day when the measures are loosened is denoted by $\delta_{3}$. After that day, the infection rate is gradually increasing

$$
b_{3}(t)=\min \left(b_{2}+\lambda\left(t-\delta_{3}\right), b_{0}\right),
$$

with $\lambda \in[0,0.03]$, while the mobility factor regains its initial value of $\theta_{0}$. 
Estimation of the Expected Information Gain The calculation of the expected utility from equation (7) is performed with Monte-Carlo integration. Samples from the prior distribution are denoted by $\boldsymbol{\vartheta}^{(i)} \sim p(\boldsymbol{\vartheta})$ and by $\widetilde{\boldsymbol{\vartheta}}^{(i)} \sim p(\widetilde{\boldsymbol{\vartheta}})$, while samples on the measurement space are denoted by $\boldsymbol{y}^{(i, j)} \sim p\left(\boldsymbol{y} \mid \boldsymbol{\vartheta}^{(i)}, \widetilde{\boldsymbol{\vartheta}}^{(i)}, \boldsymbol{s}\right)$, where $i \in\left\{1, \ldots, N_{\vartheta}\right\}$ and $j \in\left\{1, \ldots, N_{y}\right\}$. With these samples, an estimate of the expected utility is computed as

$$
\begin{aligned}
& \hat{U}(\boldsymbol{s})=\frac{1}{N_{\boldsymbol{\vartheta}} N_{\boldsymbol{y}}} \sum_{i=1}^{N_{\vartheta}} \sum_{j=1}^{N_{y}}\left[\log \left(\frac{p\left(\boldsymbol{y}^{(i, j)} \mid \boldsymbol{\vartheta}^{(i)}, \widetilde{\boldsymbol{\vartheta}}^{(i)}, \boldsymbol{s}\right)}{p\left(\boldsymbol{y}^{(i, j)} \mid \widetilde{\boldsymbol{\vartheta}}^{(i)}, \boldsymbol{s}\right)}\right)\right] \\
& p\left(\boldsymbol{y}^{(i, j)} \mid \widetilde{\boldsymbol{\vartheta}}^{(i)}, \boldsymbol{s}\right):=\frac{1}{N_{\boldsymbol{\vartheta}}} \sum_{n=1}^{N_{\vartheta}} p\left(\boldsymbol{y}^{(i, j)} \mid \boldsymbol{\vartheta}^{(n)}, \widetilde{\boldsymbol{\vartheta}}^{(i)}, \boldsymbol{s}\right) .
\end{aligned}
$$

In our implementation the samples $\boldsymbol{\vartheta}^{(i)}$ and $\widetilde{\boldsymbol{\vartheta}}^{(i)},\left(i=1, \ldots, N_{\theta}\right)$, remain the same for different values of $\boldsymbol{s}$. Thus, the model evaluations $\boldsymbol{g}\left(\boldsymbol{s}, \boldsymbol{\vartheta}^{(i)}, \widetilde{\boldsymbol{\vartheta}}^{(i)}\right)$ are only carried out once and are stored and used in the iteration process involved in the optimization. This allows to separate the computational cost of the model evaluation from the cost of computing the utility, which scales as $\mathcal{O}\left(N_{\vartheta}^{2} N_{y}\right)$.

Optimal Location and Time of Testing We define the optimal test times and locations as

$$
\boldsymbol{s}^{*}=\underset{s_{1}, \ldots, s_{M_{y}}}{\arg \max } \hat{U}(\boldsymbol{s}),
$$

where $s^{*}=\left(s_{1}^{*}, \ldots, s_{M_{y}}^{*}\right)$ with $s_{i}^{*}=\left(k_{i}^{*}, t_{i}^{*}\right)$ denote the locations $k_{i}^{*}$ and times $t_{i}^{*}$ for the optimal measurements with $i \in\left\{1, \ldots, M_{y}\right\}$. For a grid search, the associated computational cost is $\mathcal{O}\left((K T)^{M_{y}}\right)$ and thus grows exponentially with the number of tests. This curse of dimensionality is avoided by using a sequential optimization method $(23,31,32)$ to approximate the global optimum by iteratively solving

$$
s_{n}^{*}=\underset{s}{\arg \max } \hat{U}_{n}(s), \forall n=1, \ldots, M_{y},
$$

where $s=(k, t)$ is the location and time to be estimated sequentially starting with $n=1$ and

$$
\hat{U}_{n}(s)=\hat{U}(s), \quad s=\left(s_{1}^{*}, \ldots, s_{n-1}^{*}, s\right) .
$$


Following this, we define the expected information gain for testing sequence $n$ as

$$
\Delta \hat{U}_{n}(s)= \begin{cases}\hat{U}_{1}(s), & n=1 \\ \hat{U}_{n}(s)-\hat{U}_{n-1}\left(s_{n-1}^{*}\right), & n>1\end{cases}
$$

Quantification of Uncertainty A data informed prior $p(\boldsymbol{\vartheta} \mid \boldsymbol{d})$ of the model parameters $\boldsymbol{\vartheta}$ can be computed from available data $\boldsymbol{d}=\left(d_{1}, \ldots, d_{M_{d}}\right)$, collected at $M_{d}$ locations and days. Here, available data $\boldsymbol{d}$ refer to the daily number of reported infectious individuals and they are contrasted from the data $\boldsymbol{y}$ of the number of unreported infectious individuals. The latter are obtained from testing strategies at selected populations using optimal experimental design. The data is mapped via a distinct model output $f(s, \boldsymbol{\vartheta}, \widetilde{\boldsymbol{\vartheta}})$ through the following error model

$$
p\left(d_{i} \mid \boldsymbol{\vartheta}, \widetilde{\boldsymbol{\vartheta}}, \nu\right)=\mathcal{N B}\left(d_{i} \mid f\left(s_{i}, \boldsymbol{\vartheta}, \widetilde{\boldsymbol{\vartheta}}\right), \nu\right), \quad i=1, \ldots, M_{d}
$$

where $\mathcal{N B}$ is the negative binomial distribution with mean $f$ and dispersion $\nu$. Also, $s_{i}=$ $\left(k_{i}, t_{i}\right)$ is the location and time the data $d_{i}$ was collected. The choice of a different error model, compared to equation (1), is based on the assumption that the data are independent and identically distributed. Such an assumption would not be acceptable in the measurement model in equation (1), as it may result in uncorrelated measurements that can become clustered in small time intervals $(28,29)$.

The data $\boldsymbol{d}=\left(d_{1}, \ldots, d_{M_{d}}\right)$ are the daily number of reported infections per canton in Switzerland (25) which corresponds to the following model quantity

$$
f\left(s_{i}, \boldsymbol{\vartheta}, \widetilde{\boldsymbol{\vartheta}}\right):=\int_{t_{i}-0.5}^{t_{i}+0.5} \frac{\alpha}{Z} E_{k_{i}}(\tau) d \tau \approx \frac{\alpha}{Z} E_{k_{i}}\left(t_{i}\right) .
$$

The posterior distribution that will be used subsequently as a data informed prior is obtained using Bayes' theorem

$$
p(\boldsymbol{\vartheta}, \widetilde{\boldsymbol{\vartheta}} \mid \boldsymbol{d})=\frac{p(\boldsymbol{d} \mid \boldsymbol{\vartheta}, \widetilde{\boldsymbol{\vartheta}}) p(\boldsymbol{\vartheta}, \widetilde{\boldsymbol{\vartheta}})}{p(\boldsymbol{d})},
$$

and is sampled with a nested sampling algorithm (17). Note the difference to equation (6) and the optimal testing methodology, where we are interested to reduce the uncertainty in 
$p(\boldsymbol{\vartheta} \mid \boldsymbol{y}, \widetilde{\boldsymbol{\vartheta}}, \boldsymbol{s})$, which excludes the nuisance parameters $\widetilde{\boldsymbol{\vartheta}}$. For the dispersion parameter in equation (18), it is assumed that $\nu=r f\left(s_{i}, \boldsymbol{\vartheta}, \widetilde{\boldsymbol{\vartheta}}\right)$. The coefficient $r$ is unknown and included in the parameter set, where $r \sim \mathcal{U}([0,2])$.

The three inferences performed are summarized in table S5, which shows the involved model parameters in each case. The histograms for the found samples are shown in figures $\mathrm{S} 1, \mathrm{~S} 2$, and $\mathrm{S} 3$.

We remark that, using the present methodology, the inferred date for the beginning of the intervention is $\delta_{1}=22.5$, which is the $18^{\text {th }}$ of March 2020, corresponding well with the $17^{\text {th }}$ of March 2020 on which the lockdown was introduced in Switzerland (26). Moreover, we infer a significant reduction in the mobility factor, which indicates that traffic between cantons was also minimized. For the inference III we plot the fit using the inferred parameters in figure S4. The daily reported cases per canton are shown, together with the data used for the inference. 
medRxiv preprint doi: https://doi.org/10.1101/2020.07.20.20157818; this version posted July 27, 2020. The copyright holder for this preprint (which was not certified by peer review) is the author/funder, who has granted medRxiv a license to display the preprint in perpetuity. All rights reserved. No reuse allowed without permission.

\section{Figures}
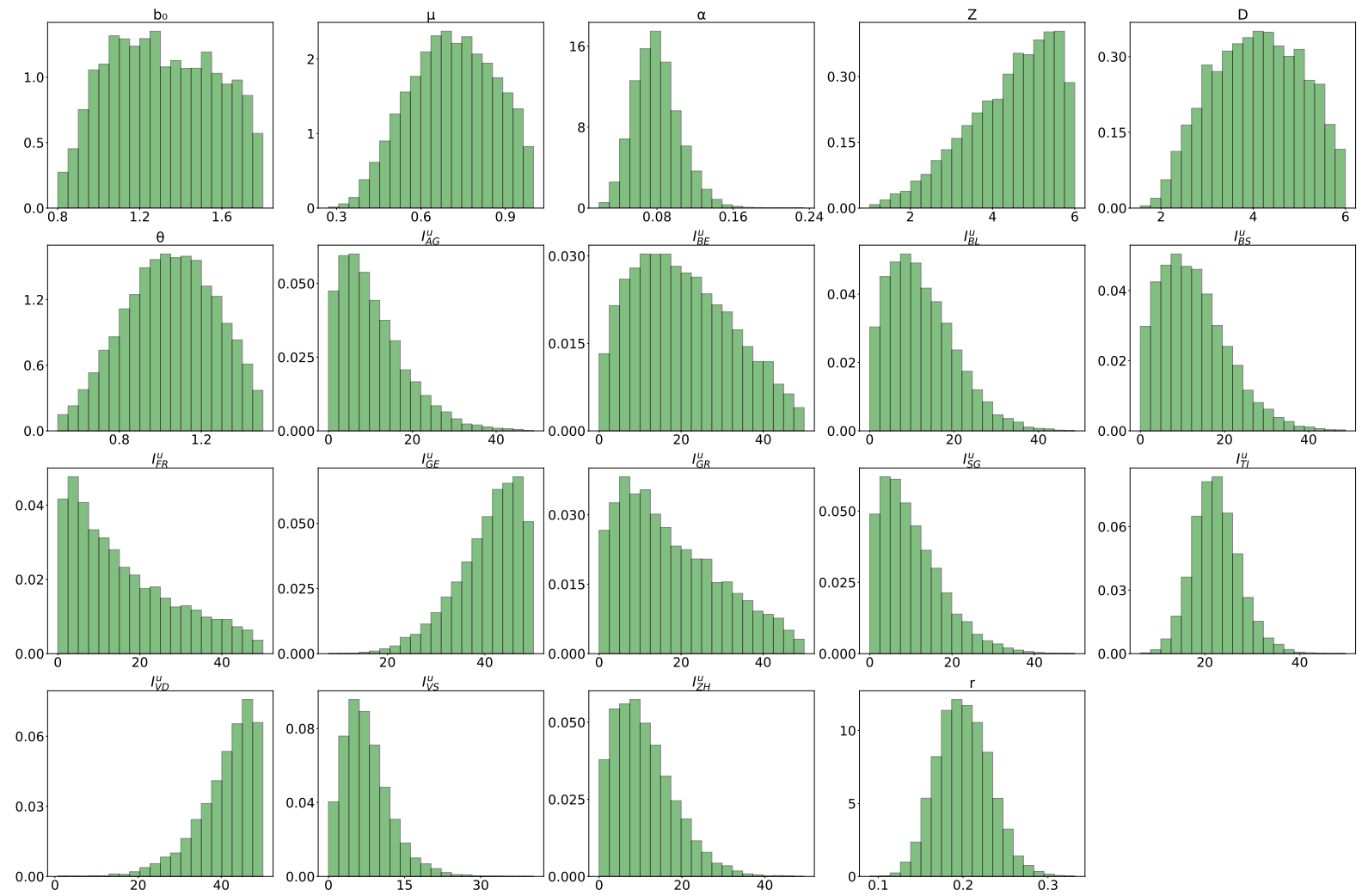

Figure S1: Marginal posterior distributions with data up to $17^{\text {th }}$ of March 2020. The used data correspond to the daily reported infectious persons in the cantons of Switzerland. The marginals with a canton label XY correspond to the initial condition $I_{\mathrm{XY}}^{u}(t=0)$ for the unreported cases in that canton. 

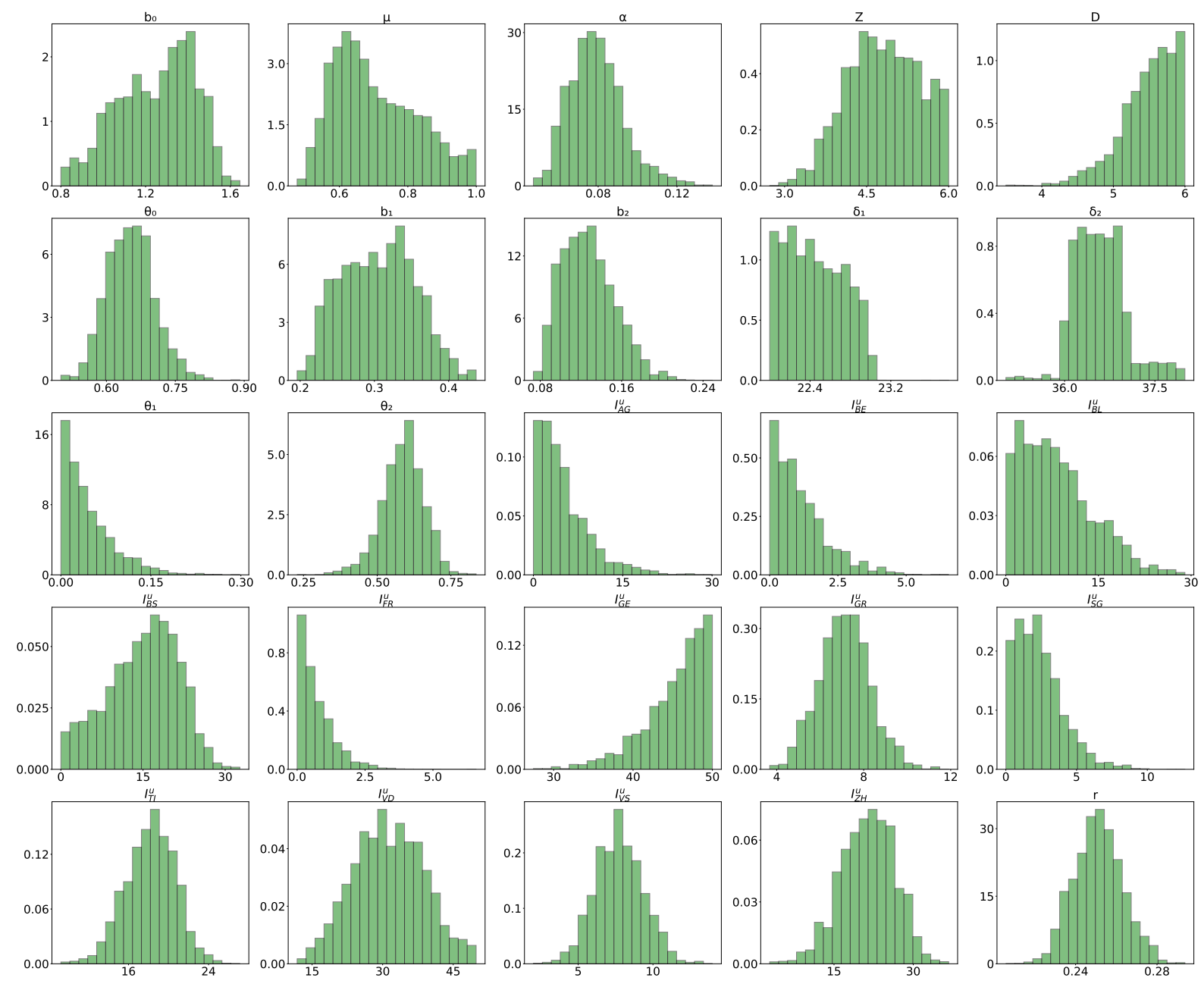

Figure S2: Marginal posterior distributions with data up to $6^{\text {th }}$ of June 2020. The used data correspond to the daily reported infectious persons in the cantons of Switzerland. The marginals with a canton label $\mathrm{XY}$ correspond to the initial condition $I_{\mathrm{XY}}^{u}(t=0)$ for the unreported cases in that canton. 

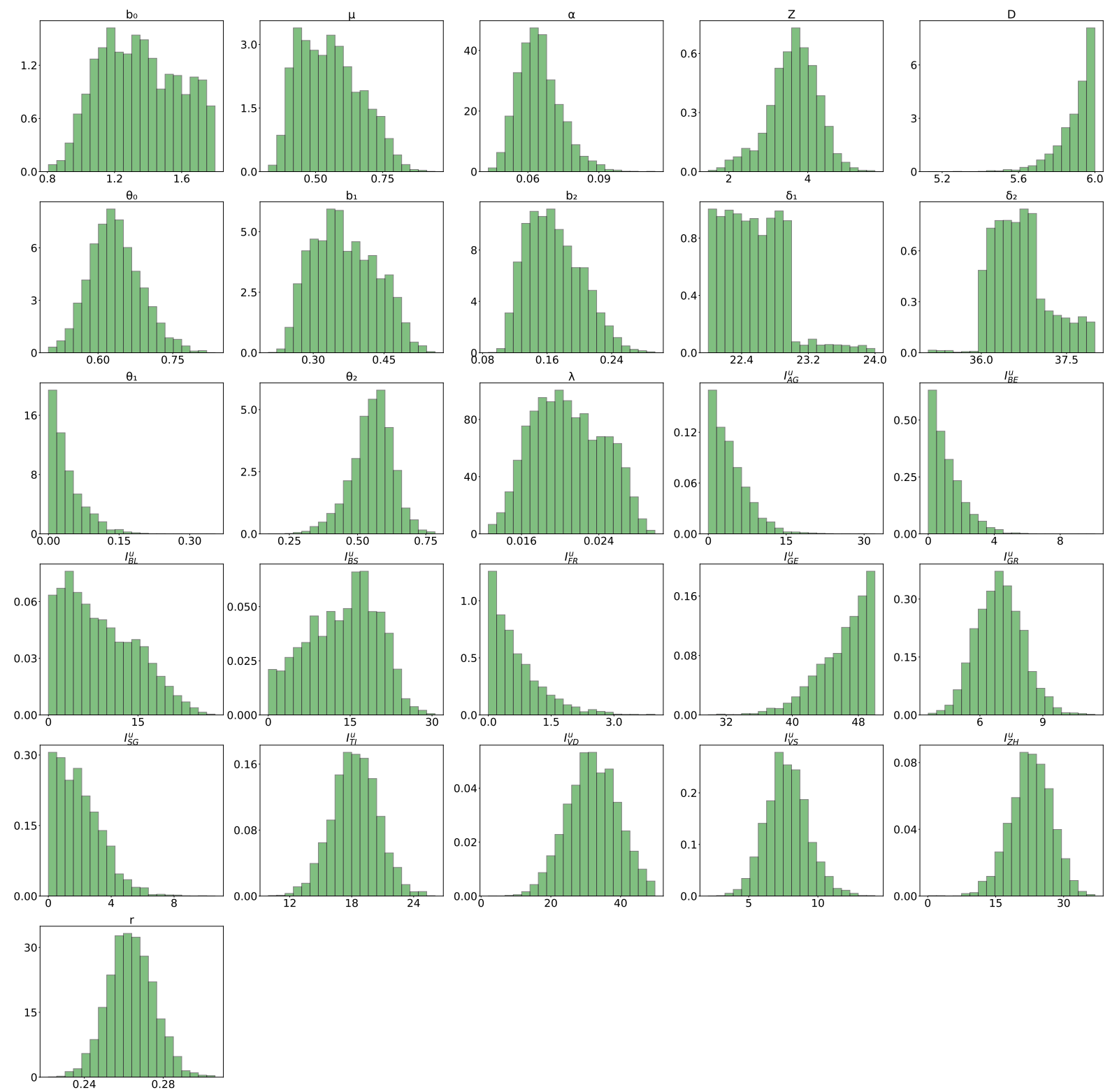

Figure S3: Marginal posterior distributions with data up to $9^{\text {th }}$ of July 2020. The used data correspond to the daily reported infectious persons in the cantons of Switzerland. The marginals with a canton label $\mathrm{XY}$ correspond to the initial condition $I_{\mathrm{XY}}^{u}(t=0)$ for the unreported cases in that canton. 

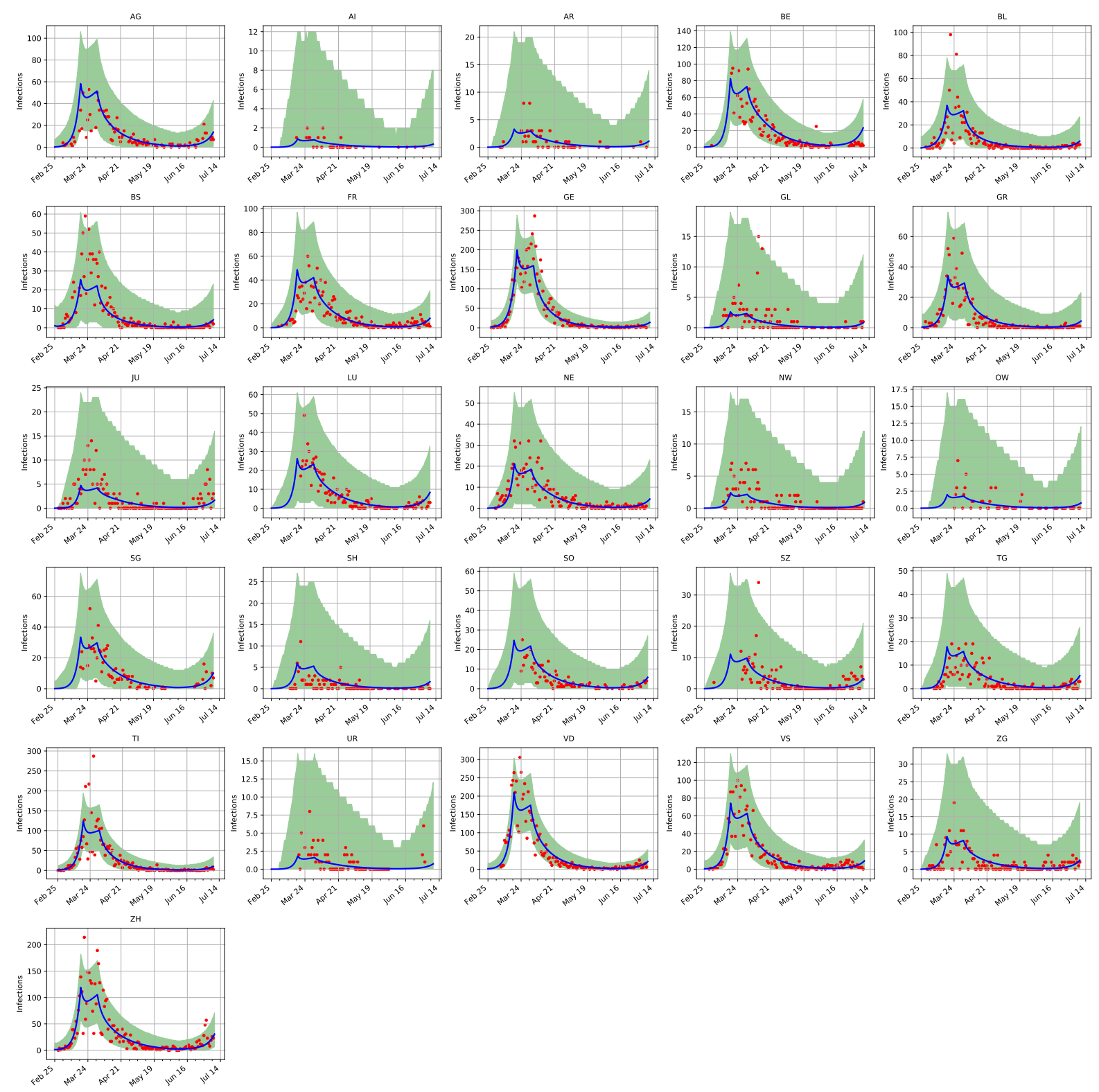

Figure S4: Maximum a-posteriori prediction with data up to $9^{\text {th }}$ of July 2020. The red points correspond to the daily reported cases per cantons and the blue curve shows the maximum aposteriori prediction. The $99 \%$ confidence interval is plotted in green and based on the sample shown in figure $\mathrm{S} 3$. 

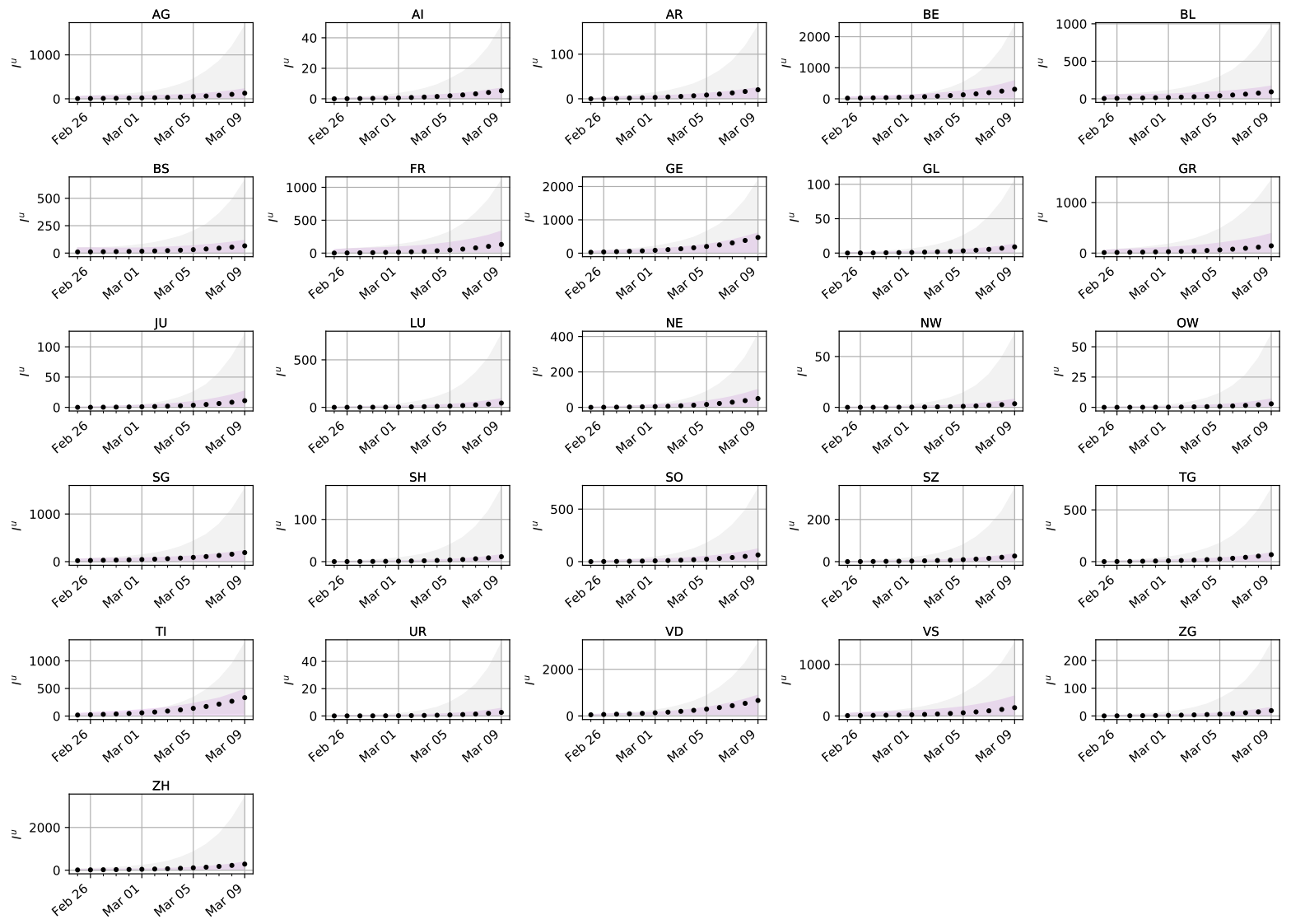

Figure S5: Comparison of prediction uncertainty per canton. The predictions are based on optimal strategies and non-specific testing for collection of data. They are also based on the $S E I^{r} I^{u} R$ model output. The error bounds show the $99 \%$ confidence intervals of the unreported infectious model output for samples of the parameters with data obtained by optimal (purple) and standard testing (gray). The black dots show the actual unreported infectious for an artificial spread in Switzerland. 

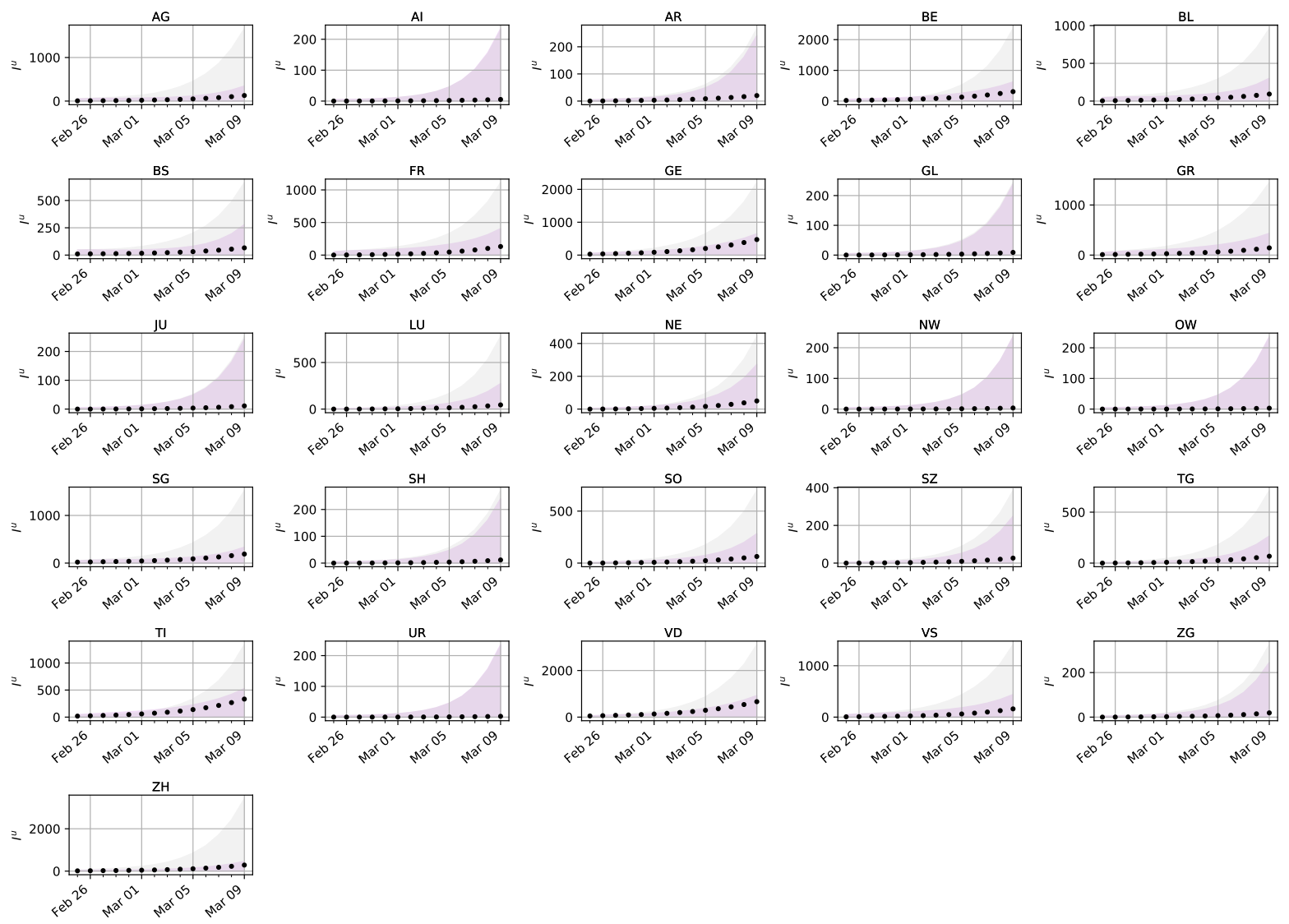

Figure S6: Comparison of propagated uncertainty per canton. The predictions are based on optimal strategies and non-specific testing. The $S E I^{r} I^{u} R$ model output with added model error for the unreported infectious is shown. The error bounds show the $99 \%$ confidence intervals of the model output with added model error for samples of the parameters with data obtained by optimal (purple) and standard testing (gray). The black dots show the actual unreported infectious for an artificial spread in Switzerland. 


\begin{tabular}{|c|c|c|c|c|}
\hline \multirow[b]{2}{*}{ Canton } & \multicolumn{4}{|c|}{ Maximum of Expected Information Gain } \\
\hline & $1^{\text {st }}$ measurement & $2^{\text {nd }}$ measurement & $3^{\text {rd }}$ measurement & $4^{\text {th }}$ measurement \\
\hline AG & $2.297(01-03)$ & $1.080(27-02)$ & $0.530(28-02)$ & $0.436(27-02)$ \\
\hline AI & $0.240(03-03)$ & $0.167(28-02)$ & $0.152(27-02)$ & $0.123(28-02)$ \\
\hline AR & $0.538(03-03)$ & $0.189(28-02)$ & $0.157(03-03)$ & $0.127(26-02)$ \\
\hline $\mathrm{BE}$ & $2.547(02-03)$ & $1.130(27-02)$ & $0.558(03-03)$ & $0.432(28-02)$ \\
\hline BL & $2.224(29-02)$ & $1.099(27-02)$ & $0.567(28-02)$ & $0.458(28-02)$ \\
\hline $\mathrm{BS}$ & $1.930(29-02)$ & $0.969(27-02)$ & $0.545(27-02)$ & $0.445(27-02)$ \\
\hline FR & $2.046(01-03)$ & $0.983(27-02)$ & $0.533(28-02)$ & $0.424(27-02)$ \\
\hline GE & $2.338(02-03)$ & $1.074(27-02)$ & $0.512(03-03)$ & $0.410(28-02)$ \\
\hline GL & $0.344(03-03)$ & $0.171(28-02)$ & $0.152(27-02)$ & $0.124(01-03)$ \\
\hline GR & $2.174(01-03)$ & $1.039(27-02)$ & $0.517(29-02)$ & $0.416(28-02)$ \\
\hline JU & $0.408(03-03)$ & $0.176(29-02)$ & $0.154(01-03)$ & $0.125(03-03)$ \\
\hline LU & $1.220(03-03)$ & $0.340(29-02)$ & $0.225(01-03)$ & $0.173(01-03)$ \\
\hline $\mathrm{NE}$ & $0.828(03-03)$ & $0.234(29-02)$ & $0.176(01-03)$ & $0.137(01-03)$ \\
\hline NW & $0.246(03-03)$ & $0.168(01-03)$ & $0.152(02-03)$ & $0.123(03-03)$ \\
\hline OW & $0.225(03-03)$ & $0.168(03-03)$ & $0.152(02-03)$ & $0.124(03-03)$ \\
\hline SG & $2.067(01-03)$ & $0.981(27-02)$ & $0.519(28-02)$ & $0.425(27-02)$ \\
\hline $\mathrm{SH}$ & $0.456(03-03)$ & $0.182(29-02)$ & $0.156(01-03)$ & $0.126(01-03)$ \\
\hline SO & $1.515(03-03)$ & $0.455(28-02)$ & $0.256(27-02)$ & $0.199(27-02)$ \\
\hline SZ & $0.785(03-03)$ & $0.221(29-02)$ & $0.167(29-02)$ & $0.134(28-02)$ \\
\hline TG & $1.214(03-03)$ & $0.334(28-02)$ & $0.209(28-02)$ & $0.163(28-02)$ \\
\hline TI & $2.362(02-03)$ & $1.077(27-02)$ & $0.516(03-03)$ & $0.409(28-02)$ \\
\hline UR & $0.210(03-03)$ & $0.167(03-03)$ & $0.152(02-03)$ & $0.124(28-02)$ \\
\hline VD & $2.666(02-03)$ & $1.233(27-02)$ & $0.594(03-03)$ & $0.314(29-02)$ \\
\hline VS & $2.254(01-03)$ & $1.061(27-02)$ & $0.514(28-02)$ & $0.417(28-02)$ \\
\hline $\mathrm{ZG}$ & $0.701(03-03)$ & $0.206(28-02)$ & $0.161(29-02)$ & $0.130(28-02)$ \\
\hline $\mathrm{ZH}$ & $2.721(02-03)$ & $1.187(27-02)$ & $0.556(28-02)$ & $0.449(27-02)$ \\
\hline
\end{tabular}

Table S1: Maximum expected information gain for outbreak of a new disease. The shown expected information gain per measurement is defined in equation (17). The corresponding optimal dates are shown in parenthesis. 


\begin{tabular}{|c|c|c|c|c|}
\hline \multirow[b]{2}{*}{ Canton } & \multicolumn{4}{|c|}{ Maximum of Expected Information Gain } \\
\hline & $1^{\text {st }}$ measurement & $2^{\text {nd }}$ measurement & $3^{\text {rd }}$ measurement & $4^{\text {th }}$ measurement \\
\hline $\mathrm{AG}$ & $2.307(30-03)$ & $1.128(17-03)$ & $0.399(30-03)$ & $0.264(30-03)$ \\
\hline AI & $0.211(30-03)$ & $0.169(17-03)$ & $0.146(21-03)$ & $0.112(26-03)$ \\
\hline AR & $0.474(30-03)$ & $0.205(17-03)$ & $0.154(29-03)$ & $0.115(29-03)$ \\
\hline $\mathrm{BE}$ & $2.927(30-03)$ & $1.738(17-03)$ & $0.691(17-03)$ & $0.459(17-03)$ \\
\hline $\mathrm{BL}$ & $1.663(30-03)$ & $0.710(17-03)$ & $0.241(17-03)$ & $0.167(17-03)$ \\
\hline $\mathrm{BS}$ & $1.359(30-03)$ & $0.518(17-03)$ & $0.196(17-03)$ & $0.140(17-03)$ \\
\hline FR & $2.149(30-03)$ & $1.093(17-03)$ & $0.256(17-03)$ & $0.176(17-03)$ \\
\hline GE & $2.825(29-03)$ & $1.760(17-03)$ & $0.483(21-03)$ & $0.327(21-03)$ \\
\hline GL & $0.364(30-03)$ & $0.183(17-03)$ & $0.149(28-03)$ & $0.114(29-03)$ \\
\hline GR & $1.783(30-03)$ & $1.256(17-03)$ & $0.754(17-03)$ & $0.222(21-03)$ \\
\hline $\mathrm{JU}$ & $0.736(30-03)$ & $0.251(17-03)$ & $0.163(30-03)$ & $0.121(30-03)$ \\
\hline $\mathrm{LU}$ & $1.830(30-03)$ & $0.738(17-03)$ & $0.316(30-03)$ & $0.210(30-03)$ \\
\hline $\mathrm{NE}$ & $1.573(30-03)$ & $0.626(17-03)$ & $0.205(17-03)$ & $0.145(17-03)$ \\
\hline NW & $0.369(30-03)$ & $0.181(17-03)$ & $0.149(30-03)$ & $0.114(29-03)$ \\
\hline OW & $0.332(30-03)$ & $0.176(17-03)$ & $0.148(30-03)$ & $0.114(29-03)$ \\
\hline SG & $2.056(30-03)$ & $1.003(17-03)$ & $0.471(17-03)$ & $0.247(17-03)$ \\
\hline $\mathrm{SH}$ & $0.625(30-03)$ & $0.215(17-03)$ & $0.158(29-03)$ & $0.119(30-03)$ \\
\hline $\mathrm{SO}$ & $1.642(30-03)$ & $0.663(17-03)$ & $0.224(30-03)$ & $0.155(30-03)$ \\
\hline SZ & $1.066(30-03)$ & $0.330(17-03)$ & $0.186(30-03)$ & $0.135(30-03)$ \\
\hline $\mathrm{TG}$ & $1.495(30-03)$ & $0.548(17-03)$ & $0.250(30-03)$ & $0.168(30-03)$ \\
\hline $\mathrm{TI}$ & $2.640(29-03)$ & $1.639(17-03)$ & $0.668(17-03)$ & $0.436(17-03)$ \\
\hline UR & $0.315(30-03)$ & $0.175(17-03)$ & $0.148(29-03)$ & $0.114(29-03)$ \\
\hline VD & $3.139(30-03)$ & $1.961(17-03)$ & $0.415(22-03)$ & $0.311(23-03)$ \\
\hline VS & $2.313(30-03)$ & $1.251(17-03)$ & $0.397(17-03)$ & $0.264(17-03)$ \\
\hline $\mathrm{ZG}$ & $0.920(30-03)$ & $0.285(17-03)$ & $0.172(30-03)$ & $0.127(30-03)$ \\
\hline $\mathrm{ZH}$ & $2.980(30-03)$ & $1.695(17-03)$ & $0.735(17-03)$ & $0.494(17-03)$ \\
\hline
\end{tabular}

Table S2: Maximum expected information gain of non-pharmaceutical interventions. The corresponding optimal dates are shown in parenthesis. 


\begin{tabular}{|c|c|c|c|c|}
\hline \multirow[b]{2}{*}{ Canton } & \multicolumn{4}{|c|}{ Maximum of Expected Information Gain } \\
\hline & $1^{\text {st }}$ measurement & $2^{\text {nd }}$ measurement & $3^{\text {rd }}$ measurement & $4^{\text {th }}$ measurement \\
\hline $\mathrm{AG}$ & $1.356(13-06)$ & $0.434(06-06)$ & $0.292(06-06)$ & $0.210(13-06)$ \\
\hline AI & $0.171(13-06)$ & $0.167(09-06)$ & $0.159(09-06)$ & $0.142(08-06)$ \\
\hline AR & $0.207(13-06)$ & $0.169(08-06)$ & $0.159(08-06)$ & $0.142(11-06)$ \\
\hline $\mathrm{BE}$ & $1.877(13-06)$ & $0.746(06-06)$ & $0.435(06-06)$ & $0.339(13-06)$ \\
\hline $\mathrm{BL}$ & $0.712(13-06)$ & $0.236(07-06)$ & $0.186(06-06)$ & $0.156(13-06)$ \\
\hline $\mathrm{BS}$ & $0.512(13-06)$ & $0.202(06-06)$ & $0.172(06-06)$ & $0.148(13-06)$ \\
\hline FR & $0.973(13-06)$ & $0.330(06-06)$ & $0.208(13-06)$ & $0.184(13-06)$ \\
\hline GE & $1.490(13-06)$ & $0.644(06-06)$ & $0.381(13-06)$ & $0.328(13-06)$ \\
\hline GL & $0.189(13-06)$ & $0.168(08-06)$ & $0.159(12-06)$ & $0.142(08-06)$ \\
\hline GR & $0.567(13-06)$ & $0.219(06-06)$ & $0.173(06-06)$ & $0.152(13-06)$ \\
\hline $\mathrm{JU}$ & $0.255(13-06)$ & $0.173(07-06)$ & $0.161(06-06)$ & $0.143(13-06)$ \\
\hline LU & $0.936(13-06)$ & $0.286(07-06)$ & $0.213(06-06)$ & $0.168(13-06)$ \\
\hline $\mathrm{NE}$ & $0.576(13-06)$ & $0.222(06-06)$ & $0.172(13-06)$ & $0.153(13-06)$ \\
\hline NW & $0.193(13-06)$ & $0.168(07-06)$ & $0.159(06-06)$ & $0.142(08-06)$ \\
\hline OW & $0.187(13-06)$ & $0.167(06-06)$ & $0.159(07-06)$ & $0.142(11-06)$ \\
\hline SG & $1.084(13-06)$ & $0.330(06-06)$ & $0.238(06-06)$ & $0.179(13-06)$ \\
\hline $\mathrm{SH}$ & $0.247(13-06)$ & $0.172(07-06)$ & $0.161(06-06)$ & $0.142(07-06)$ \\
\hline $\mathrm{SO}$ & $0.701(13-06)$ & $0.235(06-06)$ & $0.184(06-06)$ & $0.154(13-06)$ \\
\hline $\mathrm{SZ}$ & $0.403(13-06)$ & $0.187(07-06)$ & $0.167(06-06)$ & $0.145(13-06)$ \\
\hline $\mathrm{TG}$ & $0.651(13-06)$ & $0.223(06-06)$ & $0.183(06-06)$ & $0.153(13-06)$ \\
\hline $\mathrm{TI}$ & $1.241(13-06)$ & $0.539(06-06)$ & $0.367(13-06)$ & $0.322(13-06)$ \\
\hline UR & $0.186(13-06)$ & $0.167(09-06)$ & $0.158(09-06)$ & $0.142(08-06)$ \\
\hline VD & $1.881(13-06)$ & $0.873(06-06)$ & $0.505(13-06)$ & $0.443(13-06)$ \\
\hline VS & $1.138(13-06)$ & $0.420(06-06)$ & $0.256(13-06)$ & $0.223(13-06)$ \\
\hline ZG & $0.337(13-06)$ & $0.180(07-06)$ & $0.163(07-06)$ & $0.144(13-06)$ \\
\hline $\mathrm{ZH}$ & $2.092(13-06)$ & $0.862(06-06)$ & $0.592(06-06)$ & $0.276(09-06)$ \\
\hline
\end{tabular}

Table S3: Maximum expected information gain for monitoring of a second outbreak with uninformed $b_{3}$. The corresponding optimal dates are shown in parenthesis. 


\begin{tabular}{|c|c|c|c|c|}
\hline \multirow[b]{2}{*}{ Canton } & \multicolumn{4}{|c|}{ Maximum of Expected Information Gain } \\
\hline & $1^{\text {st }}$ measurement & $2^{\text {nd }}$ measurement & $3^{\text {rd }}$ measurement & $4^{\text {th }}$ measurement \\
\hline AG & $1.233(17-07)$ & $0.328(10-07)$ & $0.220(17-07)$ & $0.194(10-07)$ \\
\hline AI & $0.170(17-07)$ & $0.167(17-07)$ & $0.154(13-07)$ & $0.136(17-07)$ \\
\hline AR & $0.198(17-07)$ & $0.169(10-07)$ & $0.154(14-07)$ & $0.136(16-07)$ \\
\hline $\mathrm{BE}$ & $1.596(17-07)$ & $0.460(10-07)$ & $0.347(17-07)$ & $0.267(10-07)$ \\
\hline $\mathrm{BL}$ & $0.616(17-07)$ & $0.202(10-07)$ & $0.166(17-07)$ & $0.147(10-07)$ \\
\hline BS & $0.441(17-07)$ & $0.184(10-07)$ & $0.159(17-07)$ & $0.141(10-07)$ \\
\hline FR & $0.636(17-07)$ & $0.209(10-07)$ & $0.193(17-07)$ & $0.156(17-07)$ \\
\hline GE & $0.896(17-07)$ & $0.326(17-07)$ & $0.308(17-07)$ & $0.225(17-07)$ \\
\hline GL & $0.184(17-07)$ & $0.168(13-07)$ & $0.154(17-07)$ & $0.136(10-07)$ \\
\hline GR & $0.418(17-07)$ & $0.182(10-07)$ & $0.162(17-07)$ & $0.141(10-07)$ \\
\hline JU & $0.219(17-07)$ & $0.169(10-07)$ & $0.155(17-07)$ & $0.136(17-07)$ \\
\hline LU & $0.834(17-07)$ & $0.234(10-07)$ & $0.178(17-07)$ & $0.157(10-07)$ \\
\hline $\mathrm{NE}$ & $0.378(17-07)$ & $0.180(10-07)$ & $0.165(17-07)$ & $0.142(17-07)$ \\
\hline NW & $0.187(17-07)$ & $0.168(10-07)$ & $0.154(15-07)$ & $0.135(10-07)$ \\
\hline OW & $0.183(17-07)$ & $0.168(10-07)$ & $0.154(10-07)$ & $0.136(12-07)$ \\
\hline $\mathrm{SG}$ & $0.994(17-07)$ & $0.267(10-07)$ & $0.191(17-07)$ & $0.169(10-07)$ \\
\hline $\mathrm{SH}$ & $0.232(17-07)$ & $0.170(10-07)$ & $0.154(15-07)$ & $0.136(11-07)$ \\
\hline SO & $0.581(17-07)$ & $0.197(10-07)$ & $0.166(17-07)$ & $0.145(10-07)$ \\
\hline SZ & $0.362(17-07)$ & $0.178(10-07)$ & $0.157(10-07)$ & $0.139(10-07)$ \\
\hline TG & $0.591(17-07)$ & $0.200(10-07)$ & $0.164(17-07)$ & $0.145(10-07)$ \\
\hline TI & $0.556(17-07)$ & $0.318(17-07)$ & $0.296(17-07)$ & $0.224(17-07)$ \\
\hline UR & $0.181(17-07)$ & $0.168(11-07)$ & $0.154(16-07)$ & $0.135(16-07)$ \\
\hline VD & $1.297(17-07)$ & $0.452(17-07)$ & $0.433(17-07)$ & $0.281(10-07)$ \\
\hline VS & $0.655(17-07)$ & $0.245(17-07)$ & $0.228(17-07)$ & $0.176(17-07)$ \\
\hline ZG & $0.303(17-07)$ & $0.174(10-07)$ & $0.155(15-07)$ & $0.137(11-07)$ \\
\hline $\mathrm{ZH}$ & $1.976(17-07)$ & $0.675(10-07)$ & $0.276(14-07)$ & $0.250(13-07)$ \\
\hline
\end{tabular}

Table S4: Maximum expected information gain to monitor a second outbreak with informed $b_{3}$. The corresponding optimal dates are shown in parenthesis. 


\begin{tabular}{lllll}
\hline Parameter & Prior distribution / fixed value & Inference I & Inference II & Inference III \\
\hline$b_{0}$ & $\mathcal{U}([0.8,1.8])$ & yes & yes & yes \\
$\alpha$ & $\mathcal{U}([0.01,1])$ & yes & yes & yes \\
$\mu$ & $\mathcal{U}([0.2,1])$ & yes & yes & yes \\
$Z$ & $\mathcal{U}([1,6])$ & yes & yes & yes \\
$D$ & $\mathcal{U}([1,6])$ & yes & yes & yes \\
$\theta_{0}$ & $\mathcal{U}([0.5,1.5])$ & yes & yes & yes \\
$c_{1}$ & $\mathcal{U}([0,1])$ & no & yes & yes \\
$c_{2}$ & $\mathcal{U}([0,1])$ & no & yes & yes \\
$c_{3}$ & $\mathcal{U}([0,1])$ & no & yes & yes \\
$c_{4}$ & $\mathcal{U}([0,1])$ & no & yes & yes \\
$\delta_{1}$ & $\mathcal{U}([20,30])$ & no & yes & yes \\
$\delta_{2}$ & $\mathcal{U}([30,40])$ & no & yes & yes \\
$\delta_{3}$ & $\mathcal{H} 2$ & no & yes & yes \\
$\lambda$ & $\mathcal{U}([0,0.03])$ & no & no & yes \\
$r$ & $\mathcal{U}([0,2])$ & yes & yes & yes \\
$\boldsymbol{I}_{\text {IC }}^{u}$ & $\mathcal{U}\left([0,50]^{12}\right)$ & yes & yes & yes \\
\hline
\end{tabular}

Table S5: Parameters and prior distributions used in Bayesian inference. Here the data corresponds to the daily reported infections. In all cases, data are used from the $25^{\text {th }}$ of February 2020, when the first reported case was found in the canton of Ticino. Inference I uses data up to the day non-pharmaceutical interventions were announced (17 ${ }^{\text {th }}$ of March 2020). Inference II uses data up to the day measures were relaxed ( $6^{\text {th }}$ of June 2020). Inference III uses data up to the $9^{\text {th }}$ of July 2020. The choice of prior distributions is consistent with the choice found in (14); the ranges used in our study are slightly extended.

\section{References}

1. D. Adam, Special report: The simulations driving the world's response to COVID-19. Nature 580, 316 (2020).

2. N. Zhu et al., A Novel Coronavirus from Patients with Pneumonia in China, 2019. New England Journal of Medicine 382, 727 (2020). 
medRxiv preprint doi: https://doi.org/10.1101/2020.07.20.20157818; this version posted July 27, 2020. The copyright holder for this preprint (which was not certified by peer review) is the author/funder, who has granted medRxiv a license to display the preprint in perpetuity.

All rights reserved. No reuse allowed without permission.

3. A. Du Toit, Outbreak of a novel coronavirus. Nature Reviews Microbiology 18, 123 (2020).

4. J. Ioannidis, The infection fatality rate of COVID-19 inferred from seroprevalence data. medRxiv (2020).

5. J. Haushofer C. J. E. Metcalf, Which interventions work best in a pandemic? Science 368, 1063 (2020).

6. M. Esbin et al., Overcoming the bottleneck to widespread testing: A rapid review of nucleic acid testing approaches for COVID-19 detection. RNA 26, rna.076232.120 (2020).

7. J. Eberhardt, N. Breuckmann C. Eberhardt, Multi-Stage Group Testing Improves Efficiency of Large-Scale COVID-19 Screening. Journal of Clinical Virology 128, 104382 (2020).

8. N. Sinnott-Armstrong, D. Klein B. Hickey, Evaluation of Group Testing for SARS-CoV-2 RNA. medRxiv (2020).

9. J. Dehning et al., Inferring change points in the spread of COVID-19 reveals the effectiveness of interventions. Science (2020).

10. K. Chaloner I. Verdinelli, Bayesian Experimental Design: A Review. Statistical Science 10, $273(1995)$.

11. X. Huan Y. M. Marzouk, Simulation-based optimal Bayesian experimental design for nonlinear systems. Journal of Computational Physics 232, 288 (2013).

12. E. G. Ryan, C. C. Drovandi, J. M. McGree A. N. Pettitt, A Review of Modern Computational Algorithms for Bayesian Optimal Design. International Statistical Review 84, 128 (2016). 
medRxiv preprint doi: https://doi.org/10.1101/2020.07.20.20157818; this version posted July 27, 2020. The copyright holder for this preprint (which was not certified by peer review) is the author/funder, who has granted medRxiv a license to display the preprint in perpetuity.

All rights reserved. No reuse allowed without permission.

13. A. E. Gongora et al., A Bayesian experimental autonomous researcher for mechanical design. Science Advances 6 (2020).

14. R. Li et al., Substantial undocumented infection facilitates the rapid dissemination of novel coronavirus (SARS-CoV-2). Science 368, 489 (2020).

15. E. T. Jaynes, Probability Theory: The Logic of Science (Cambridge University Press, 2003).

16. P. Karnakov et al., Data driven inference of the reproduction number for COVID-19 before and after interventions for 51 European countries. Swiss Medical Weekly (2020).

17. J. S. Speagle, Dynesty: a dynamic nested sampling package for estimating Bayesian posteriors and evidences. Monthly Notices of the Royal Astronomical Society 493, 3132-3158 (2020).

18. D. Lindley, On a measure of the information provided by an experiment. Annals of mathematical statistics 27, 986 (1956).

19. E. Karni D. Schmeidler (Elsevier, 1991), vol. 4 of Handbook of Mathematical Economics, pp. $1763-1831$.

20. K. J. Ryan, Estimating Expected Information Gains for Experimental Designs With Application to the Random Fatigue-Limit Model. Journal of Computational and Graphical Statistics 12, 585 (2003).

21. P. Tsilifis, R. G. Ghanem P. Hajali, Efficient Bayesian Experimentation Using an Expected Information Gain Lower Bound. SIAM/ASA Journal on Uncertainty Quantification 5, 30 (2017).

22. J. Tolles T. Luong, Modeling Epidemics With Compartmental Models. JAMA 323, 2515 (2020). 
medRxiv preprint doi: https://doi.org/10.1101/2020.07.20.20157818; this version posted July 27, 2020. The copyright holder for this preprint (which was not certified by peer review) is the author/funder, who has granted medRxiv a license to display the preprint in perpetuity.

All rights reserved. No reuse allowed without permission.

23. C. Papadimitriou, Optimal sensor placement methodology for parametric identification of structural systems. Journal of Sound and Vibration 278, 923 (2004).

24. Y. Liu, A. A. Gayle, A. Wilder-Smith J. Rocklöv, The reproductive number of COVID-19 is higher compared to SARS coronavirus. Journal of Travel Medicine 27 (2020).

25. Statistisches Amt Kanton Zürich, SARS-CoV-2 Cases communicated by Swiss Cantons and Principality of Liechtenstein (FL), https://github.com/openZH/covid_19 (2020). Online.

26. Bundesamt für Gesundheit, Neues Coronavirus, https://www.bag.admin.ch/bag/de/home/ krankheiten/ausbrueche-epidemien-pandemien/aktuelle-ausbrueche-epidemien/novelcov.html (2020). Online.

27. D. Berry, Bayesian clinical trials. Nature Reviews Drug Discovery 5,27-36,2006

28. C. Papadimitriou G. Lombaert, The effect of prediction error correlation on optimal sensor placement in structural dynamics. Mechanical Systems and Signal Processing 28, 105 (2012).

29. E. Simoen, C. Papadimitriou G. Lombaert, On prediction error correlation in Bayesian model updating. Journal of Sound and Vibration 332, 4136 (2013).

30. Bundesamt für Statistik, Employed persons by commune of residence and work, https://www.bfs.admin.ch/bfsstatic/dam/assets/8507281/master (2014). Online.

31. S. Verma et al. , Optimal sensor placement for artificial swimmers. J. Fluid Mech., 884, (2019).

32. P. Weber et al. , Optimal flow sensing for schooling swimmers. Biomimetics, 5, 1, (2020). 